Dinh et al. 2018

\title{
Multiscale spatio-temporal patterns of boat noise on U.S. Virgin Island coral reefs
}

Jason P. Dinh ${ }^{\mathrm{a}}$, Justin J. Suca ${ }^{\mathrm{b}, \mathrm{c}}$, Ashlee Lillis ${ }^{\mathrm{b}}$, Amy Apprill $^{\mathrm{d}}$, Joel Llopiz ${ }^{\mathrm{b}}$, T. Aran Mooney ${ }^{\mathrm{b}^{*}}$

aDuke University, Department of Biology

${ }^{\text {b} W o o d s ~ H o l e ~ O c e a n o g r a p h i c ~ I n s t i t u t i o n, ~ B i o l o g y ~ D e p a r t m e n t ~}$

${ }^{c}$ Massachusetts Institute of Technology, MIT/WHOI Joint Program in Oceanography

${ }^{\mathrm{d} W o o d s ~ H o l e ~ O c e a n o g r a p h i c ~ I n s t i t u t i o n, ~ M a r i n e ~ C h e m i s t r y ~ \& ~ G e o c h e m i s t r y ~ D e p a r t m e n t ~}$

\section{*Corresponding author}

Email address: amooney@whoi.edu (T.A. Mooney)

Mailing Address: 266 Woods Hole Road, Woods Hole, MA, 02543

Declarations of interest: none

Keywords: bioacoustics, noise pollution, soundscapes, ecoacoustics, coral reefs 
Dinh et al. 2018

\begin{abstract}
Sound-sensitive organisms are abundant on coral reefs. Accordingly, experiments suggest that boat noise could elicit adverse effects on coral reef organisms. Yet, there are few data quantifying boat noise prevalence on coral reefs. We use long-term passive acoustic recordings at nine coral reefs and one sandy comparison site in a marine protected area to quantify spatio-temporal variation in boat noise and its effect on the soundscape. Boat noise was most common at reefs with high coral cover and fish density, and temporal patterns reflected patterns of human activity. Boat noise significantly increased low-frequency sound levels at the monitored sites. With boat noise present, the peak frequencies of the natural soundscape shifted from higher frequencies to the lower frequencies frequently used in fish communication. Taken together, the spectral overlap between boat noise and fish communication and the elevated boat detections on reefs with biological densities raises concern for coral reef organisms.
\end{abstract}

\title{
1. Introduction
}


Dinh et al. 2018

Coral reefs host some of the highest diversity of life per unit area on Earth. About onequarter to one-third of all marine species live in coral reefs (Knowlton et al., 2010; Plaisance et al., 2011; Reaka-Kudla, 1997). Reefs provide essential ecosystem services such as shoreline protection and carbon dioxide uptake (reviewed in Moberg \& Folke, 1999). They also provide substantial economic value associated with tourism, fisheries, and the aquarium trade (Moberg \& Folke, 1999; Spalding et al., 2017). Yet in recent decades, myriad stressors such as overfishing, ocean warming, disease, and acidification have driven coral reefs into global decline (Hughes et al., 2018; McClenachan et al., 2017; Tsounis \& Edmunds, 2017). In addition to these classically studied stressors, there is increasing awareness that the resilience of reefs and basic ecological processes on coral reefs are threatened by anthropogenic noise pollution (e.g. McCormick et al., 2018; Simpson et al., 2016).

Acoustic signals are used by many coral reef organisms because of the efficient propagation of sound in water (Au \& Hastings, 2008; Myrberg, 1981). In healthy coral reefs with diverse biological communities, these soniferous organisms create a biophony comprising of fish choruses and the sounds of invertebrates such as hermit crabs and snapping shrimps (Freeman et al., 2014; Kaplan et al, 2015). These animals use sound for ecologically vital behaviors such as larval orientation (Leis et al., 2003; Radford et al. 2011; Simpson et al., 2008), agonistic territoriality (e.g. Herberholz \& Schmitz, 1998), and mate attraction (Myrberg et al., 1986).

With this growing awareness of reef acoustic ecology, there is a corresponding understanding that noise from vessels may impact key behaviors and have substantial physiological effects on coral reef organisms (Slabbekoorn et al., 2010). For example, acoustic masking occurs when the presence of one noise increases the detection threshold of another (Clark et al., 2009). In coral reefs, boat noise masks acoustic cues and disrupts orientation behavior in settlement-stage coral reef fish larvae (Holles et al., 2013; Simpson et al., 2016). 
Dinh et al. 2018

Furthermore, evidence suggests that noise from small motor vessels induces physiological stress responses in coral reef fishes. For instance, exposure to boat noise was associated with an increase in metabolic rate in Ambon damselfish (Simpson et al., 2016) and an increase in heart rate of staghorn damselfish embryos (Jain-Schlaepfer et al., 2018).

Perhaps most alarmingly, acoustic disturbance from boats may impose direct consequences on individual fitness and induce mortality in certain coral reef organisms. For instance, in situ playback of boat noise has been shown to increase embryonic mortality in sea hares and increase predation rates of settlement-stage Ambon damselfish (Nedelec et al., 2014; Simpson et al., 2016). Recent evidence suggests that boat noise could also have more indirect and latent consequences on individual fitness. For example, boat noise has been shown to decrease offspring fitness by impairing parental care in a brooding reef fish, the spiny chromis (Nedelec et al., 2017). Exposure to outboard motor noise during critical learning periods has also been shown to impair predator-learning behavior and decrease subsequent responses to predators in juvenile Ambon damselfish (Ferrari et al., 2018).

While several studies have suggested that boat noise may affect essential biological functions in coral reef organisms, little is known about the pervasiveness of this stressor. The actual levels, occurrence rates, and reef-based variation of boat noise in coral reefs are rarely reported. Several studies have suggested that passive acoustic recordings are a useful means to monitor and quantify boat activity, but this has been on a limited scale (just a few reefs and relatively short timeframes) (Kaplan \& Mooney, 2015; Lammers et al., 2008). Furthermore, while noise predictions and propagation studies have long been conducted in open ocean environments (e.g., cetsound.org) (Wenz, 1962), the physically complex shallow water environments make noise propagation modeling and predicting noise levels on reefs more challenging, thereby limiting noise predictions that are comparable to open ocean environments. 
Dinh et al. 2018

Thus, multiple in situ noise measurements become vital as we seek to understand acoustic patterns and pervasiveness of noise pollution on coral reefs.

Here, we utilized passive acoustic monitoring to quantify boat activity at nine coral reefs and one sandy shore comparison site off the island of St. John, U.S. Virgin Islands (USVI) over the course of one year. The coral reef sites are assigned the following numerical codes by which they will be referred hereafter, with site number increasing from west to east: Ditliff Point (1), Cocoloba Cay (2), Joel's Shoal (3), White Point (4), Europa Bay (5), Tektite (6), Yawzi Point (7), Booby Rock (8), and Ram Head (9) (Figure 1; Table 1). Reef Bay is an off-reef sandy bottom comparison site and will be referred to as Site S. St. John is home to the USVI National Park, a popular tourist destination, and many visitors charter small boats to visit local coral reefs. We described the spatio-temporal patterns of boat noise at each site on diel, weekly, and seasonal scales. By comparing the relative prevalence of boat noise at each site to surveys of benthic cover and fish communities, we sought to better understand the potential drivers of boat noise. Furthermore, we evaluated the effects of boat noise on the local soundscape by calculating the sound pressure level (SPL) in two separate acoustic bands: one predominantly used by fish, the other dominated by snapping shrimp. We quantified the peak acoustic frequency defined by the frequency with the highest acoustic power to evaluate how the soundscape changed in the presence of boat noise. By providing occurrence patterns and spectral consequences of boat noise, this study provides valuable baseline data that can inform management, monitoring, and experimental methodology.

\section{Methods}

\subsection{Acoustic Data Collection}


Dinh et al. 2018

We deployed passive acoustic recorders (SoundTrap ST300, Ocean Instruments NZ, Inc.) at nine shallow coral reef sites and one sandy-shore control site (all $7-12 \mathrm{~m}$ depth) along the southern shore of St. John, U.S. Virgin Islands from May 2016 - July 2017 (48 kHz sampling frequency) (Figure 1A). Acoustic recorders were attached $\sim 0.75 \mathrm{~m}$ above the seafloor to a rebar stake using hose clamps and cable ties, with the omnidirectional hydrophone facing the water surface (Figure 1B). Recording units were programmed on a 10\% duty cycle and collected oneminute recordings every ten minutes. At four times throughout the study (June 2016, August 2016, October 2016 and March 2017), acoustic recorders were removed for 1 - 3 days in order to offload data and recharge batteries and were then redeployed.

We conducted all analyses in Matlab 9.2 (MathWorks, Inc., Natick, MA). We calibrated recordings according to each hydrophone's sensitivity which was provided by the manufacturer. Spectrograms for each 1-minute recording were generated using a 16384-point FFT in 0.25second windows with $25 \%$ overlap between contiguous windows. The average power spectrum for each recording was estimated using Welch's method (Hanning window, non-overlapping 0.5second averages) (Welch, 1967).

We visually inspected spectrograms and average power spectra to identify recordings containing boat noise. Visual identification was based on broadband high intensity sound levels (Kaplan \& Mooney, 2015; Kaplan et al., 2016). Boat noise usually caused substantial changes to soundscapes and were easily detectable by visual inspection of spectrograms (Figure 2). If visual identification was ambiguous, we aurally audited to confirm or reject the presence of boat noise. We binned each recording by hour, day, and month, and we calculated the net percentage of recordings containing boat noise for each site within these bins.

For each 1-minute audio file, we calculated the low-frequency root-mean-square SPL (SPL $\left.\mathrm{rms}_{\mathrm{rm}}\right)(50-1500 \mathrm{~Hz})$, the high-frequency $\mathrm{SPL}_{\mathrm{rms}}(2 \mathrm{kHz}-20 \mathrm{kHz})$, the overall $\mathrm{SPL}_{\mathrm{rms}}$, and 
Dinh et al. 2018

the frequency with the highest acoustic power, herein called the peak frequency. The lowfrequency band was selected due to its association with fish communication, and the highfrequency band was selected to assess sound generated from snapping shrimp (Kaplan \& Mooney, 2015; Kaplan et al., 2015). The low-frequency band is also highly influenced by boat noise as well as geophonic sounds such as wind and waves. Thus, to determine natural diel patterns of sound pressure level at our sites, we considered only files without boat noise.

\subsection{Benthic and Fish Surveys}

We conducted benthic visual point surveys for soft corals, hard corals, algae, and substratum cover along six 10-m transects at each site from 7 June - 10 June 2016 and again from 17 July - 24 July 2017. Benthic cover at the point directly below each transect was recorded every 10 centimeters. Cover was classified as dead coral (bleached, newly dead, or dead), algae (crustose coralline algae, cyanobacteria, turf algae, or macroalgae), invertebrate (aggressive invertebrate or other invertebrate), live hard coral (identified to genus), live soft coral (identified to genus), or substratum (pavement, rubble, or sand). We calculated coral cover for each survey by dividing the total number of points identified as hard coral by the total number of points surveyed at each site ( $n=600$ per survey). The arithmetic mean of coral cover in the 2016 and 2017 surveys is herein called the average coral cover. The total number of hard coral genera present at each site is herein called the genus richness $(\mathrm{R})$.

We conducted four 30-m SCUBA video transects at each site between 7 June - 10 June 2016 and again between 23 July - 26 July 2017 to assess reef fish abundance and diversity. Videos were recorded on an Olympus PEN Lite E-PL5 camera. Attached to the camera's underwater housing and in the field of view was a $1 \mathrm{~m}$ long section of PVC with a $25 \mathrm{~cm}$ cross bar located $50 \mathrm{~cm}$ from the camera lens and a $50 \mathrm{~cm}$ cross bar located $1 \mathrm{~m}$ from the camera lens, 
Dinh et al. 2018

and the cross bars were marked at $5 \mathrm{~cm}$ increments. This setup assisted with estimating the width of the swath to be examined and for the estimation of fish size. At the sandy site (S), fish were rarely seen and thus a camera was not employed. Instead, the occasional fish was counted and identified by the diver and recorded underwater.

At each reef site, transects began in the vicinity of the acoustic recorder with transect bearings being haphazardly chosen. Bearings were restricted to those that largely covered reef structure to avoid surveys over large portions of sand. A diver swam along slowly holding the camera system as close to but above the reef, remaining parallel to the reef structure while at the same time laying out the transect tape. This was repeated for a total of four transects at each location.

We analyzed video transects on a high-resolution monitor to maximize capability and confidence of reef fish identification. We randomly selected three transects for analysis if all four transects contained suitable footage and no equipment malfunctions occurred. The three suitable transects were used otherwise. All fish observed in the video were enumerated and identified to lowest possible taxonomic unit. Fish sizes were estimated and grouped into the following size classes: $0-5 \mathrm{~cm}, 6-10 \mathrm{~cm}, 11-20 \mathrm{~cm}, 21-30 \mathrm{~cm}, 31-40 \mathrm{~cm}$, and $>40 \mathrm{~cm}$. We calculated total abundance and species richness of reef fishes at each. Species richness (R) was determined as the total number of reef fish species present at each reef.

At each site, we calculated Shannon-Weiner diversity indices $(\mathrm{H})$ on a genus level for corals and species level for fishes using the following formula:

$$
H=-\sum_{i=1}^{R} p_{i} * \ln \left(p_{i}\right)
$$

where $\mathrm{p}$ represents the proportion of total taxa that taxon $\mathrm{i}$ represents and $\mathrm{R}$ represents the genus richness for corals or species richness for fishes. $\mathrm{H}$ is the diversity term with larger values 
Dinh et al. 2018

representing a more diverse community. We calculated equitability of Shannon-Weiner indices to compare fish and coral diversities across sites using the following formula:

$$
\mathrm{E}_{\mathrm{x}}=\frac{\mathrm{H}_{\mathrm{x}}}{\ln (\mathrm{R})}
$$

where $\mathrm{H}_{\mathrm{x}}$ is the Shannon-Wiener index at site $\mathrm{x}, \mathrm{R}$ is the genus richness for corals or species richness for fishes at site $\mathrm{x}$, and $\mathrm{E}_{\mathrm{x}}$ is the equitability at site $\mathrm{x}$ (Pielou, 1966). Larger values of equitability represent more diverse communities.

\subsection{Statistical Analyses}

We conducted statistical analyses using the R programming language (RStudio Inc., Boston, MA). We modeled temporal variation in boat noise using binomial generalized additive mixed models (GAMMs) in the R package 'mgcv' (Wood, 2018). We removed recordings collected while our team was conducting experiments in the field from analysis of diel patterns to account for the influence of daily experiments conducted by our group. Because the samples for daily analysis were different than the samples for yearly and weekly analysis, we fitted two GAMMS: one using weekday and month as fixed effects with random slopes for weekday and month within each site, and another using hour of day as a fixed effect and hour of day within each site as a nested random effect (Chen, 2000; Hastie \& Tibshirani, 1990). Recordings from May, June, and July contained files from 2016 and 2017. For these months, we considered recordings from 2016 and 2017 together because GAMMs with cubic cyclic spines account for the cyclical nature of monthly variation.

We compared spatial variation of boat noise occurrence across sites using a Pearson's chi-squared test for independence. We used ordinary least-squares linear regression modeling to identify relationships between the percentage of recordings containing boat noise at a site and the coral cover, coral diversity, fish density, and fish diversity at that site. 
Dinh et al. 2018

To determine the acoustic effects of boat noise, we calculated median values and quantiles for SPL $\mathrm{rms}_{\mathrm{r}}$, low-frequency SPL $\mathrm{rms}_{\mathrm{rm}}(50-1500 \mathrm{~Hz})$, high-frequency SPL $\mathrm{L}_{\mathrm{rms}}(20-200$ $\mathrm{Hz}$ ), and peak frequency at each site. We binned these metrics by site and presence of boat noise. For each site, we compared the median values of these metrics in files with and without boat noise using a Mann-Whitney U test. A post-hoc Bonferroni correction was applied wherever multiple comparisons were made to correct for family-wise error rate.

\section{Results}

\subsection{Boat Noise Acoustic Characteristics}

Recordings with boat noise exhibited greater sound levels in the low-frequency band $(<1500 \mathrm{~Hz})$ compared to files without boat noise (Figure 3b). This difference was as high as 40 $\mathrm{dB}$ re $1 \mu \mathrm{Pa}^{2} \cdot \mathrm{Hz}^{-1}$ (Figure 2; Supplementary Material 1). Peak frequencies in recordings with boat noise were significantly lower than recordings of the natural soundscapes, dropping from $3000-6000 \mathrm{~Hz}$ to $100-500 \mathrm{~Hz}$ (Figure 3d).

Overall SPL and low-frequency SPL were significantly higher in recordings with boat noise at all sites (Figure $3 \mathrm{a}$ and $\mathrm{b}$ ). The contribution of motorboats also caused significant differences in high-frequency SPL at six of the ten sites (Figure 3c; Supplementary Material 2). At the other four sites, the median high-frequency SPL $\mathrm{Sms}_{\mathrm{rms}}$ was lower in files with boat noise (Figure 3c; Supplementary Material 2). Despite statistical significance at six sites, the actual differences in median high-frequency $\mathrm{SPL}_{\mathrm{rms}}$ in the presence of boat noise were small (i.e. within $1 \mathrm{~dB}$ ) and likely an artifact of high statistical power from large sample size.

\subsection{Spatial and Temporal Patterns}


Dinh et al. 2018

Boat noise was a nearly daily occurrence at most sites. Boat noise was observed in over $90 \%$ of days at half the sites and in at least $70 \%$ of the days at another 4 sites (Table 1 ). Only Reef 5 had relatively few boat detection days at $<30 \%$. The overall percentage of files with boat noise at each site varied between 1 and 5\% (Figure 4). Boats were most commonly detected at Reef 3 and least commonly detected at Reef $5\left(X^{2}=9480, p<0.0005\right)$ (Figure 4; Supplementary Material 3). The percentage of recordings containing boat noise at a given site was positively correlated with average coral cover and fish density. There was no significant correlation between the percentage of recordings with boat noise and coral or fish diversity (Figure 5).

Smoothing estimators were statistically significant for fixed effects of hour, day of week, and month $(\mathrm{p}<0.05)$ (Figure 6; Table 2). Boat noise was lowest at night between 20:00 and 04:00 (Figures 7 and 8). Occurrences increased starkly at dawn (ca 06:00) and steadily increased throughout daylight hours until late afternoon for most sites (except Reef 7), with peaks in boat activity typically occurring at 15:00 and 16:00 (Figures 7 and 8; Table 3).

Clear crepuscular peaks in SPL were noted in the high-frequency band (Figure 8). Dawn and dusk peaks were also notable in the low-frequency acoustic band, but SPL generally remained elevated (compared to nighttime levels) throughout daylight hours. The elevations in the low frequencies were shorter in duration, with sound levels usually decreasing shortly after the respective 18:00 peak. Contrarily, SPL elevations in the higher frequencies exhibited more gradual transitions.

Boat noise occurrence also varied among days of the week, with peaks between Thursday and Sunday (Table 3; Supplementary Material 4). However, weekly patterns were weaker than diel and annual patterns (Figure 6). On an annual scale, boat noise was least common during the summer months (June - September) (Figure 6; Supplementary Material 5). Boat noise detections increased through autumn and early winter before peaking in late winter and early spring (ca. 
Dinh et al. 2018

January - March), coinciding with the general patterns in the tourist season (Figure 6;

Supplementary Material 5).

\section{Discussion}

Here, we found that boat noise was a near-daily occurrence on the coral reefs studied in the U.S. Virgin Islands National Park (Table 1). This was especially true at sites with the highest density of fishes and corals (e.g. Reefs 3 and 6), where boats were detected on $97-98 \%$ of recording days (Table 1). We observed clear temporal patterns with peaks in boat noise in the early afternoon, weekends, and in the late winter through early spring (Figure 6). The presence of boat noise was associated with increases in overall SPL (Figure 3a). The strongest increases arose in low frequencies, reflecting an overall change in the soundscape induced by boat noise (Figure 3b). These lower frequencies are used by many fishes and some invertebrates for acoustic communication (Freeman et al., 2014; Kaplan et al., 2015; Myrberg, 1981; Tricas \& Boyle, 2014). The percentage of files with boat noise also varied among reefs, with detections being most common at sites with high coral cover and fish density (Figure 5). Taken together, the spatial, temporal, and acoustic patterns presented here suggest that boat noise could be an acoustic stressor on coral reefs, especially on those with a high density of fishes and coral cover.

We do not have visual observation data that could indicate the likely sources of this noise. We suspect that this noise often comes from boats supporting recreational SCUBA diving and snorkeling activity at coral reefs. Two sites with high occurrence rates of boat noise (Reefs 6 and 8) have SCUBA moorings, supporting the suggestion that elevated boat noise is at least partly due to recreational diving. However, the exact sources of boat noise remain speculative until further study. We encourage future studies to use synchronous audio-visual recordings or 
Dinh et al. 2018

vessel transects to visually identify vessel types and activities associated with boat noise on coral reefs.

If SCUBA activity indeed is the primary driver of boat noise on the monitored coral reefs, then the detection of elevated boat noise at coral reefs with the highest density of biota suggests that recreational diving may impose an acoustic stressor on the ecosystems with the greatest number of organisms at risk. Further, if this pattern is indeed the result of recreational diving, monitoring such noise could be an indicator of coincident SCUBA-related stressors on coral reefs, including coral fragmentation (Hawkins \& Roberts, 1993), sediment deposition (Zakai \& Chadwick-Furman, 2002), and pathogen exposure (Lamb et al., 2014). Again, a future study utilizing synchronous or contemporaneous audio-visual observations is required to confirm that SCUBA activity is the primary source of boat noise before these applications can be appropriately developed.

Anchoring is not allowed in the USVI National Park. Thus it is unlikely that sites without short-term "day" moorings (8 of our 10 sites; though two of these sites, Reefs 7 and 9, are in close proximity to overnight moorings) are subject to high levels of diver-vessel associated boat noise. Therefore, several other contributing factors such as benthic complexity, depth, and distance offshore may have affected the variation in boat noise rates between sites. For example, boat noise was more prevalent at the sandy site $S$, than at 6 of the 9 coral reefs. This is likely a function of improved propagation at sandy sites compared to complex benthic systems like coral reefs. As a sandy shore site, S also tends to have lower levels of biological sounds (unpublished data), and boats may be more easily detected in these quieter conditions.

Further, boats were most commonly detected at Reef 3 . This site is slightly further offshore and in deeper water than the other sites where boats traveling the coastline are more likely to pass. Additionally, Reefs 6 and 7 are located at the entrance to Greater Lameshur Bay 
Dinh et al. 2018

that contains U.S. National Parks Service overnight moorings, which may have influenced boat noise occurrences (Figure 1). Reefs 1, 2, and 9 are also located near inlets where boat moorings are present (Figure 1). However, many, if not most, of the moored boats were sailboats and catamarans (pers. obs.), which are likely quieter boats than motor vessels. It therefore remains unlikely that boats at overnight moorings are the sole contributors to elevated boat noise at nearby sites.

Occurrences of boat noise were elevated during daylight hours, reflecting levels of human activity. We found that patterns in boat noise occurrences overlap temporally with patterns in fish calling, although the exact patterns of the two sounds did not match precisely. We used natural low-frequency sound pressure levels to approximate diel patterns in fish calls at our recorded sites. In this analysis, we calculated sound pressure levels using only recordings without boat noise, thereby separating the influence of boat noise and fish chorusing on sound pressure levels. Low-frequency sound pressure levels were elevated during daylight hours compared to nighttime hours, and crepuscular peaks were present at most sites, consistent with previous studies (Kaplan et al., 2015) (Figure 8). Thus, while peaks in boat noise were not exactly coincident with crepuscular peaks in ambient fish calls and natural sound levels, elevated presence of boats during daylight hours when fishes are commonly calling may still pose a potential temporal risk for acoustic masking.

The presence of boat noise increased SPL particularly in lower frequencies, reflecting the dominant frequencies of the noise from the vessel engines. Yet, the SPL measured in this study may still underestimate the acoustic levels experienced by coral reef organisms because many fishes and invertebrates detect sound primarily in the form of particle motion (e.g. Montgomery et al., 2006; Radford et al., 2016). Acoustic particle motion of boat noise exceeds SPL within 5 meters from the noise source (Wahlberg et al., 2008). Thus, while SPL measurements are 
Dinh et al. 2018

sufficient for identification of boat noise, future studies that quantify the particle motion associated with boat noise in shallow-water environments may be more appropriate to fully understand the extent to which boat noise may affect coral reef organisms.

Coincident with occurrences of boat noise, peak frequencies switched from the higher, snapping shrimp-dominated bandwidth (ca. $3-6 \mathrm{kHz}$ ) to the fish communication bandwidth (ca $100-500)$, suggesting that boat noise changes the patterns of the overall soundscape. Reefs 4 and 8 were the two exceptions where naturally occurring soundscapes were dominated by lowfrequency sounds. The anomaly at these two sites is likely the result of increased wave action, wind, and other geophonic sources that predominantly affect frequencies lower than $1 \mathrm{kHz}$ (Wenz 1962).

Intense acoustic energy at frequencies below $1 \mathrm{kHz}$ raises concerns for reef fishes, whose hearing thresholds and sound production lie within this range (Maruska et al., 2007; Myrberg et al., 1986; Wright et al. 2005). The frequency overlap between boat noise and natural noise raises concerns for acoustic masking in coral reef fishes, who use sound for ecological functions including courtship (Myrberg et al., 1986), individual recognition (Myrberg \& Riggio, 1985), and larval settlement (Radford et al., 2011). While acoustic masking has not been welldocumented in adult coral reef fishes, it has been demonstrated in several non-reef fishes (e.g. Codarin et al., 2009; Vasconcelos et al., 2007). One concern is that individual fish sound signals are often not very high in amplitude (Amorim, 2006), making them vulnerable to masking. Formal calculations of masking are beyond the scope of this study. However, given the frequency range of reef fish communication and the temporal patterns of boat noise presented here, we believe that coral reef fishes are prone to masking, and we highly encourage future study to quantitively assess the effects of boat noise on communication space in coral reef fishes. 
Dinh et al. 2018

The chronic and repeated exposures to boat noise may also have implications on the behavior, physiology, and sensory neurobiology of coral reef organisms. Some evidence suggests that coral reef fishes may desensitize to boat noise during short-term continuous exposure (Holmes et al., 2017). However, long-term desensitization here is unlikely given the temporal unpredictability and the acoustic variability between boat noise occurrences. Even within a national park, which can be some of our most protected marine areas, daily exposure to boat noise still poses a chronic stressor to resident coral reef fishes and invertebrates. The need to include anthropogenic noise in ecosystem management plans becomes increasingly clear given the frequent detections of boat noise presented here and the burgeoning body of scientific evidence elucidating the biological consequences of small vessel noise. Noise from small boats can have lethal ecological and physiological effects on coral reef organisms, with known consequences ranging from larval recruitment (Holles et al., 2013; Simpson et al., 2016) and cognition (Ferrari et al., 2018) to behavior (Holmes et al., 2017; McCormick et al., 2018; Nedelec et al., 2017), and embryonic development (Jain-Schlaepfer et al., 2018; Nedelec et al., 2014). The data presented here suggest there is potential for these effects to already be manifesting, even within national parks, and will continue to exacerbate with increasing demand for tourist activities on coral reefs (Gil et al., 2015; Piggott-McKellar \& McNamara, 2017). The U.S. National Parks Service (NPS) addresses threats associated with anthropogenic noise through the Natural Sounds and Night Skies Division ("Natural Sounds (U.S. National Park Service)"). However, most efforts outlined in this program specifically address terrestrial ecosystems and noise pollution from overflights. The data presented here suggest that natural sounds of aquatic national parks are also at risk to noise pollution and may face frequent noise events. Thus, we encourage the expansion of U.S. NPS acoustic monitoring and prevention initiatives to include marine and coastal national parks. Boat noise is easily detectable and 
Dinh et al. 2018

potentially preventable, and management of this ubiquitous pollutant could abate a chronic stressor in an ecosystem that is already facing myriad other chemical and physical threats.

Our results show that acoustic monitoring data can quantify boat activity and noise exposure on relatively fine spatial (individual reefs) and temporal (hourly) scales. This tool is particularly useful in remote areas where visual inspection is infeasible or not supported and in marine protected areas where boat activity is potentially restricted. These methods could be used to inform ecosystem managers regarding not only noise exposure, but also potentially fishing and harvesting in closed areas, diving in restricted areas, and other usage violations in marine protected areas. Furthermore, the data presented here provide valuable baseline information elucidating the extent and patterns of anthropogenic noise in coral reefs. These baseline data can be used to assess current and future levels of noise pollution and can be applied to inform methodology of laboratory studies.

\section{Acknowledgements:}

This research was funded by the National Science Foundation Biological Oceanography Program (award OCE-1536782) and the WHOI Summer Student Fellowship Program. The fieldwork was conducted under National Park Service Scientific Research and Collecting Permits VIIS-2016-SCI-0018 and VIIS-2017-SCI-0019. We would like to thank Max Kaplan, Ian Jones, Jessica Perelman, Alexis Earl, Jessica Dehn, Cynthia Becker, Laura Weber, and Rod Catanach for their assistance in the field and the staff volunteers at the Virgin Islands Environmental Resource Station (VIERS) for their logistical assistance and support. We would also like to thank the Academic Programs Office at the Woods Hole Oceanographic Institution (WHOI). We also appreciate the input of two anonymous reviewers. Their constructive comments greatly improved the overall quality of our manuscript. 
Dinh et al. 2018

\section{References:}

Amorim, M. C. P. (2006). Diversity of sound production in fish. In Communication in Fishes (Vol. 1, pp. 71-105). Science Publishers.

Au, W. W. L., \& Hastings, M. C. (2008). Principles of Marine Bioacoustics (2008 edition). New York: Springer.

Chen, C. (2000). Generalized additive mixed models. Communications in Statistics - Theory and Methods, 29(5-6), 1257-1271. https://doi.org/10.1080/03610920008832543

Clark, C. W., Ellison, W. T., Southall, B. L., Hatch, L., Van Parijs, S. M., Frankel, A., \& Ponirakis, D. (2009). Acoustic masking in marine ecosystems: intuitions, analysis, and implication. Marine Ecology Progress Series, 395, 201-222.

Codarin, A., Wysocki, L. E., Ladich, F., \& Picciulin, M. (2009). Effects of ambient and boat noise on hearing and communication in three fish species living in a marine protected area (Miramare, Italy). Marine Pollution Bulletin, 58(12), 1880-1887. https://doi.org/10.1016/j.marpolbul.2009.07.011

Ferrari, M. C. O., McCormick, M. I., Meekan, M. G., Simpson, S. D., Nedelec, S. L., \& Chivers, D. P. (2018). School is out on noisy reefs: the effect of boat noise on predator learning and survival of juvenile coral reef fishes. Proc. R. Soc. B, 285(1871), 20180033. https://doi.org/10.1098/rspb.2018.0033

Freeman, S. E., Rohwer, F. L., D’Spain, G. L., Friedlander, A. M., Gregg, A. K., Sandin, S. A., \& Buckingham, M. J. (2014). The origins of ambient biological sound from coral reef ecosystems in the Line Islands archipelago. The Journal of the Acoustical Society of America, 135(4), 1775-1788. https://doi.org/10.1121/1.4865922 
Dinh et al. 2018

Gil, M. A., Renfro, B., Figueroa-Zavala, B., Penié, I., \& Dunton, K. H. (2015). Rapid tourism growth and declining coral reefs in Akumal, Mexico. Marine Biology, 162(11), 22252233. https://doi.org/10.1007/s00227-015-2748-z

Hastie, T. J., \& Tibshirani, R. J. (1990). Generalized Additive Models. CRC Press.

Hawkins, J. P., \& Roberts, C. M. (1993). Effects of Recreational Scuba Diving on Coral Reefs: Trampling on Reef-Flat Communities. Journal of Applied Ecology, 30(1), 25-30. https://doi.org/10.2307/2404267

Herberholz, J., \& Schmitz, B. (1998). Role of Mechanosensory Stimuli in Intraspecific Agonistic Encounters of the Snapping Shrimp (Alpheus heterochaelis). The Biological Bulletin, 195(2), 156-167. https://doi.org/10.2307/1542823

Holles, S., Simpson, S., D., Radford, A. N., \& Lecchini, D. (2013). Boat noise disrupts orientation behaviour in a coral reef fish. Marine Ecology Progress Series, 485, 295-300.

Holmes, L. J., McWilliam, J., Ferrari, M. C. O., \& McCormick, M. I. (2017). Juvenile damselfish are affected but desensitize to small motor boat noise. Journal of Experimental Marine Biology and Ecology, 494, 63-68.

https://doi.org/10.1016/j.jembe.2017.05.009

Hughes, T. P., Anderson, K. D., Connolly, S. R., Heron, S. F., Kerry, J. T., Lough, J. M., ... Wilson, S. K. (2018). Spatial and temporal patterns of mass bleaching of corals in the Anthropocene. Science, 359(6371), 80-83. https://doi.org/10.1126/science.aan8048

Jain-Schlaepfer, S., Fakan, E., Rummer, J. L., Simpson, S. D., \& McCormick, M. I. (2018). Impact of motorboats on fish embryos depends on engine type. Conservation Physiology, 6(1). https://doi.org/10.1093/conphys/coy014

Kaplan, M. B., \& Mooney, T. A. (2015). Ambient noise and temporal patterns of boat activity in the US Virgin Islands National Park. Marine Pollution Bulletin, 98(1-2), 221-228. 
Dinh et al. 2018

Kaplan, M. B., Mooney, T. A., Lammers, M. O., \& Zang, E. (2016). Temporal and spatial variability in vessel noise on tropical coral reefs. Proceedings of Meetings on Acoustics. Retrieved from http://asa.scitation.org.proxy.lib.duke.edu/doi/10.1121/2.0000250

Kaplan, M. B., Mooney, T. A., Partan, J., \& Solow, A. R. (2015). Coral reef species assemblages are associated with ambient soundscapes. Marine Ecology Progress Series, 533, 93-107. https://doi.org/10.3354/meps11382

Knowlton, N., Brainard, R. E., Fisher, R., Moews, M., Plaisance, L., \& Caley, M. J. (2010). Coral Reef Biodiversity. In Life in the World's Oceans (pp. 65-78). Wiley-Blackwell. https://doi.org/10.1002/9781444325508.ch4

Lamb, J. B., True, J. D., Piromvaragorn, S., \& Willis, B. L. (2014). Scuba diving damage and intensity of tourist activities increases coral disease prevalence. Biological Conservation, 178(Supplement C), 88-96. https://doi.org/10.1016/j.biocon.2014.06.027

Lammers, M. O., Brainard, R. E., Au, W. W. L., Mooney, T. A., \& Wong, K. B. (2008). An ecological acoustic recorder (EAR) for long-term monitoring of biological and anthropogenic sounds on coral reefs and other marine habitats. The Journal of the Acoustical Society of America, 123(3), 1720-1728. https://doi.org/10.1121/1.2836780

Leis, J. M., Carson-Ewart, B. M., Hay, A. C., \& Cato, D. H. (2003). Coral-reef sounds enable nocturnal navigation by some reef-fish larvae in some places and at some times. Journal of Fish Biology, 63(3), 724-737. https://doi.org/10.1046/j.1095-8649.2003.00182.x

Maruska, K. P., Boyle, K. S., Dewan, L. R., \& Tricas, T. C. (2007). Sound production and spectral hearing sensitivity in the Hawaiian sergeant damselfish, Abudefduf abdominalis. Journal of Experimental Biology, 210(22), 3990-4004.

https://doi.org/10.1242/jeb.004390 
Dinh et al. 2018

McClenachan, L., O’Connor, G., Neal, B. P., Pandolfi, J. M., \& Jackson, J. B. C. (2017). Ghost reefs: Nautical charts document large spatial scale of coral reef loss over 240 years. Science Advances, 3(9), e1603155. https://doi.org/10.1126/sciadv.1603155

McCormick, M. I., Allan, B. J. M., Harding, H., \& Simpson, S. D. (2018). Boat noise impacts risk assessment in a coral reef fish but effects depend on engine type. Scientific Reports, 8(1), 3847. https://doi.org/10.1038/s41598-018-22104-3

Moberg, F., \& Folke, C. (1999). Ecological goods and services of coral reef ecosystems. Ecological Economics, 29(2), 215-233. https://doi.org/10.1016/S0921-8009(99)00009-9

Montgomery, J. C., Jeffs, A., Simpson, S. D., Meekan, M., \& Tindle, C. (2006). Sound as an Orientation Cue for the Pelagic Larvae of Reef Fishes and Decapod Crustaceans. In B.-A. in M. Biology (Ed.) (Vol. 51, pp. 143-196). Academic Press.

https://doi.org/10.1016/S0065-2881(06)51003-X

Myrberg, A. A. (1981). Sound Communication and Interception in Fishes. In Hearing and Sound Communication in Fishes (pp. 395-426). Springer, New York, NY. https://doi.org/10.1007/978-1-4615-7186-5_20

Myrberg, A. A., Mohler, M., \& Catala, J. D. (1986). Sound production by males of a coral reef fish (Pomacentrus partitus): its significance to females. Animal Behaviour, 34(3), 913923. https://doi.org/10.1016/S0003-3472(86)80077-X

Myrberg, A. A., \& Riggio, R. J. (1985). Acoustically mediated individual recognition by a coral reef fish (Pomacentrus partitus). Animal Behaviour, 33(2), 411-416. https://doi.org/10.1016/S0003-3472(85)80065-8

Natural Sounds (U.S. National Park Service). (n.d.). Retrieved May 16, 2018, from https://www.nps.gov/subjects/sound/index.htm 
Dinh et al. 2018

Nedelec, S. L., Radford, A. N., Pearl, L., Nedelec, B., McCormick, M. I., Meekan, M. G., \& Simpson, S. D. (2017). Motorboat noise impacts parental behaviour and offspring survival in a reef fish. Proc. R. Soc. B, 284(1856), 20170143. https://doi.org/10.1098/rspb.2017.0143

Nedelec, S. L., Radford, A. N., Simpson, S. D., Nedelec, B., Lecchini, D., \& Mills, S. C. (2014). Anthropogenic noise playback impairs embryonic development and increases mortality in a marine invertebrate. Scientific Reports, 4. https://doi.org/10.1038/srep05891

Pielou, E. C. (1966). The measurement of diversity in different types of biological collections. Journal of Theoretical Biology, 13, 131-144. https://doi.org/10.1016/00225193(66)90013-0

Piggott-McKellar, A. E., \& McNamara, K. E. (2017). Last chance tourism and the Great Barrier Reef. Journal of Sustainable Tourism, 25(3), 397-415. https://doi.org/10.1080/09669582.2016.1213849

Plaisance, L., Caley, M. J., Brainard, R. E., \& Knowlton, N. (2011). The Diversity of Coral Reefs: What Are We Missing? PLOS ONE, 6(10), e25026. https://doi.org/10.1371/journal.pone.0025026

Radford, C. A., Stanley, J. A., Simpson, S. D., \& Jeffs, A. G. (2011). Juvenile coral reef fish use sound to locate habitats. Coral Reefs, 30(2), 295-305. https://doi.org/10.1007/s00338010-0710-6

Radford, Craig A., Tay, K., \& Goeritz, M. L. (2016). Hearing in the paddle crab, Ovalipes catharus. Proceedings of Meetings on Acoustics, 27(1), 010013. https://doi.org/10.1121/2.0000259

Reaka-Kudla, M. L. (1997). Global Biodiversity of Coral Reefs. In Biodiversity II : understanding and protecting our biological resources ([Rev ed.], pp. 83-108). 
Dinh et al. 2018

Washington, D.C. : Joseph Henry Press. Retrieved from

https://trove.nla.gov.au/version/46514246

Simpson, S. D., Meekan, M. G., Jeffs, A., Montgomery, J. C., \& McCauley, R. D. (2008). Settlement-stage coral reef fish prefer the higher-frequency invertebrate-generated audible component of reef noise. Animal Behaviour, 75(6), 1861-1868. https://doi.org/10.1016/j.anbehav.2007.11.004

Simpson, Stephen D., Radford, A. N., Holles, S., Ferarri, M. C. O., Chivers, D. P., McCormick, M. I., \& Meekan, M. G. (2016). Small-Boat Noise Impacts Natural Settlement Behavior of Coral Reef Fish Larvae. Advances in Experimental Medicine and Biology, 875, 10411048. https://doi.org/10.1007/978-1-4939-2981-8_129

Simpson, Stephen D., Radford, A. N., Nedelec, S. L., Ferrari, M. C. O., Chivers, D. P., McCormick, M. I., \& Meekan, M. G. (2016). Anthropogenic noise increases fish mortality by predation. Nature Communications, 7, 10544.

https://doi.org/10.1038/ncomms10544

Slabbekoorn, H., Bouton, N., van Opzeeland, I., Coers, A., ten Cate, C., \& Popper, A. N. (2010). A noisy spring: the impact of globally rising underwater sound levels on fish. Trends in Ecology \& Evolution, 25(7), 419-427. https://doi.org/10.1016/j.tree.2010.04.005

Spalding, M., Burke, L., Wood, S., Ashpole, J., Hutchinson, J., \& Ermgassen, P. zu. (2017). Mapping the global value and distribution of coral reef tourism. Marine Policy, 82, 104 113.

Tricas, T. C., \& Boyle, K. S. (2014). Acoustic behaviors in Hawaiian coral reef fish communities. Marine Ecology Progress Series, 511, 1-16. https://doi.org/10.3354/meps10930 
Dinh et al. 2018

Tsounis, G., \& Edmunds, P. J. (2017). Three decades of coral reef community dynamics in St. John, USVI: a contrast of scleractinians and octocorals. Ecosphere, 8(1), e01646. https://doi.org/10.1002/ecs2.1646

Vasconcelos, R. O., Amorim, M. C. P., \& Ladich, F. (2007). Effects of ship noise on the detectability of communication signals in the Lusitanian toadfish. Journal of Experimental Biology, 210(12), 2104-2112. https://doi.org/10.1242/jeb.004317

Wahlberg, M., Schack, H. B., Wilson, M., Bejder, L., \& Madsen, P. T. (2008). Particle Acceleration Noise Generated by Boats. Bioacoustics, 17(1-3), 148-150. https://doi.org/10.1080/09524622.2008.9753797

Welch, P. (1967). The use of fast Fourier transform for the estimation of power spectra: A method based on time averaging over short, modified periodograms. IEEE Transactions on Audio and Electroacoustics, 15(2), 70-73. https://doi.org/10.1109/TAU.1967.1161901

Wenz, G. M. (1962). Acoustic Ambient Noise in the Ocean: Spectra and Sources. The Journal of the Acoustical Society of America, 34(12), 1936-1956. https://doi.org/10.1121/1.1909155

Wood, S. (2018). mgcv: Mixed GAM Computation Vehicle with Automatic Smoothness Estimation (Version 1.8-23). Retrieved from https://CRAN.R-project.org/package=mgcv

Wright, K. J., Higgs, D. M., Belanger, A. J., \& Leis, J. M. (2005). Auditory and olfactory abilities of pre-settlement larvae and post-settlement juveniles of a coral reef damselfish (Pisces: Pomacentridae). Marine Biology, 147(6), 1425-1434. https://doi.org/10.1007/s00227-005-0028-z

Zakai, D., \& Chadwick-Furman, N. E. (2002). Impacts of intensive recreational diving on reef corals at Eilat, northern Red Sea. Biological Conservation, 105(2), 179-187. https://doi.org/10.1016/S0006-3207(01)00181-1 
Dinh et al. 2018

Tables and Figures: 
Dinh et al. 2018

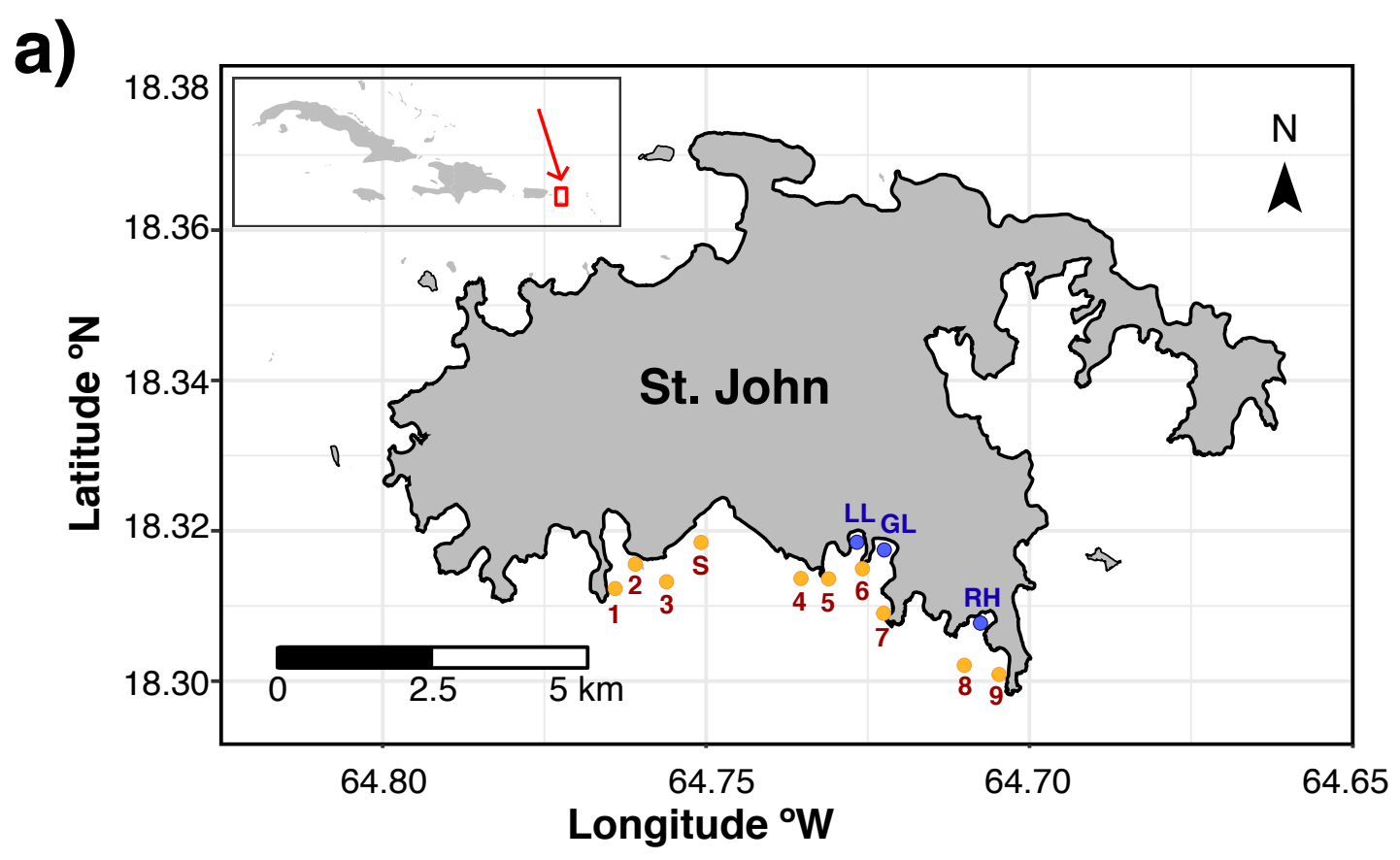

b)

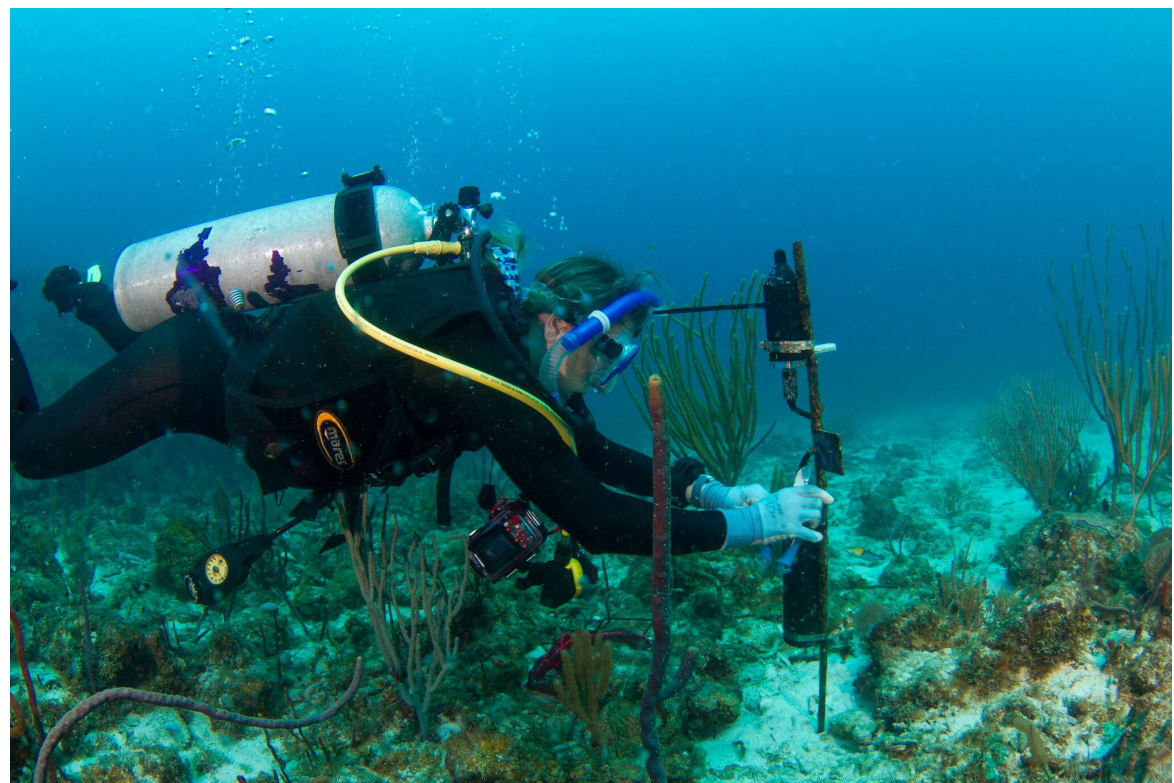

Figure 1: Field sites at St. John and deployment of hydrophones. (a) Map displaying locations of ten acoustically monitored sites off the southern shore of St. John, U.S. Virgin Islands (orange points). The red box indicates the location of St. John within the Caribbean. Blue points indicate mooring sites at Little Lameshur Bay (LL), Greater Lameshur Bay (GL), and Ram Head (RH). (b) Example of passive acoustic recorder deployment. Recording units were 
Dinh et al. 2018

attached to a rebar stake and deployed with the omnidirectional hydrophone facing the sea surface. 


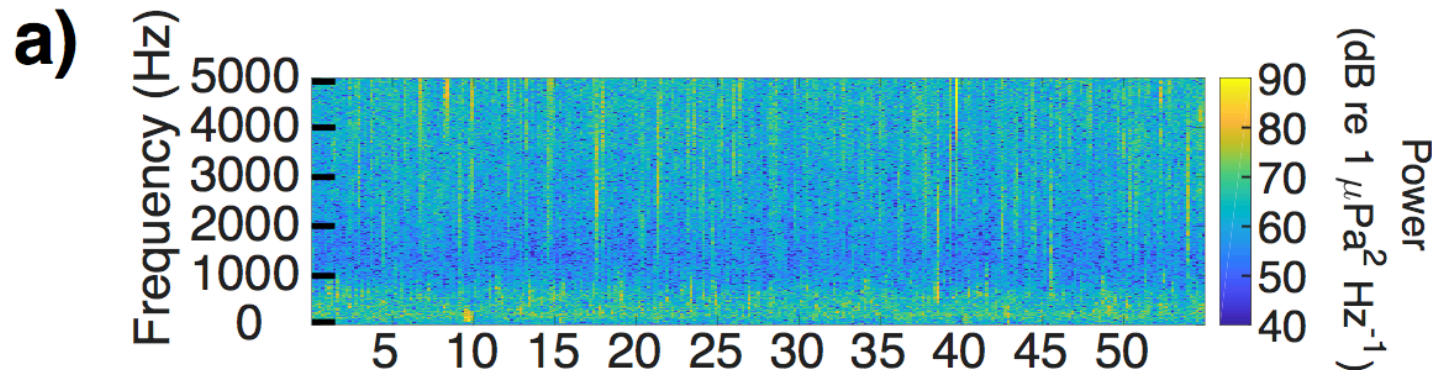

b) $\widehat{N} \quad$ Time (sec)

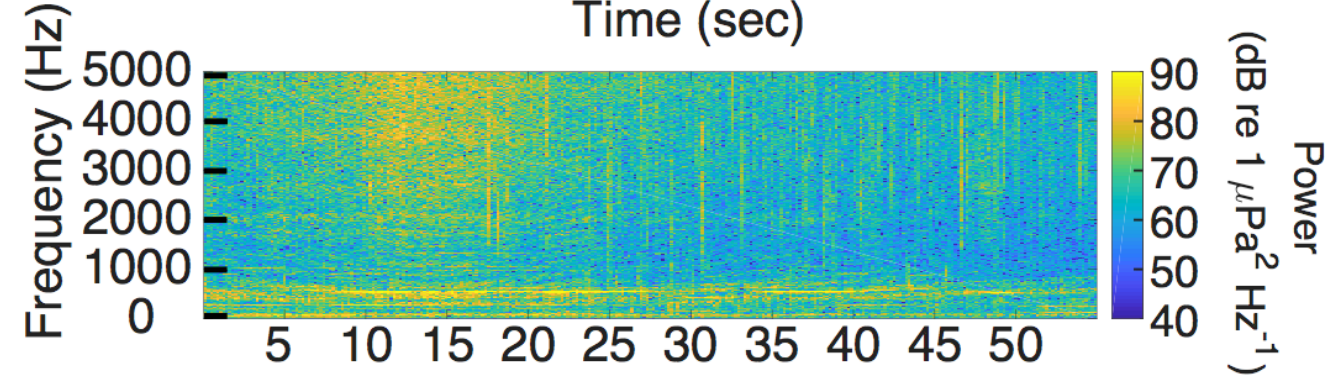

Time (sec)

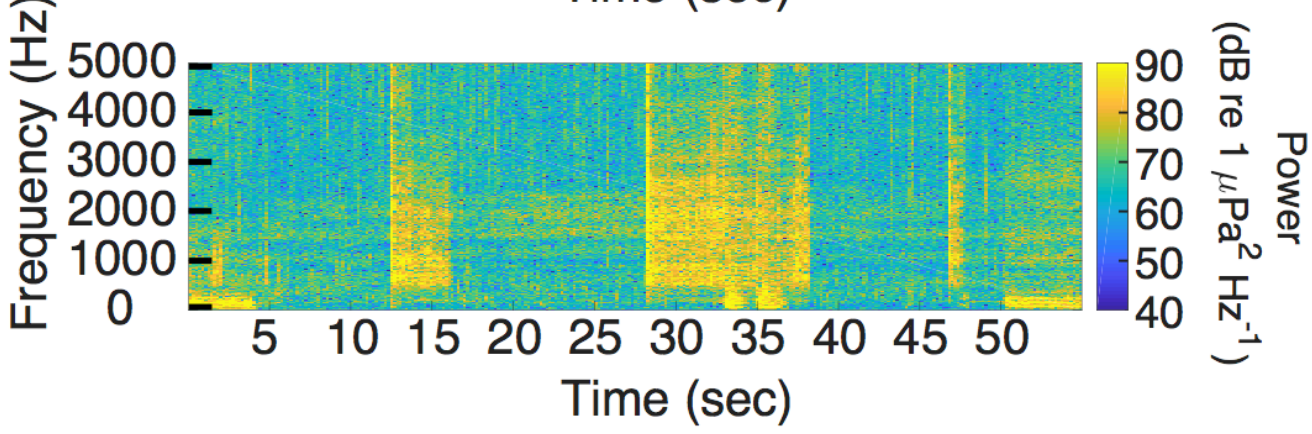

d)

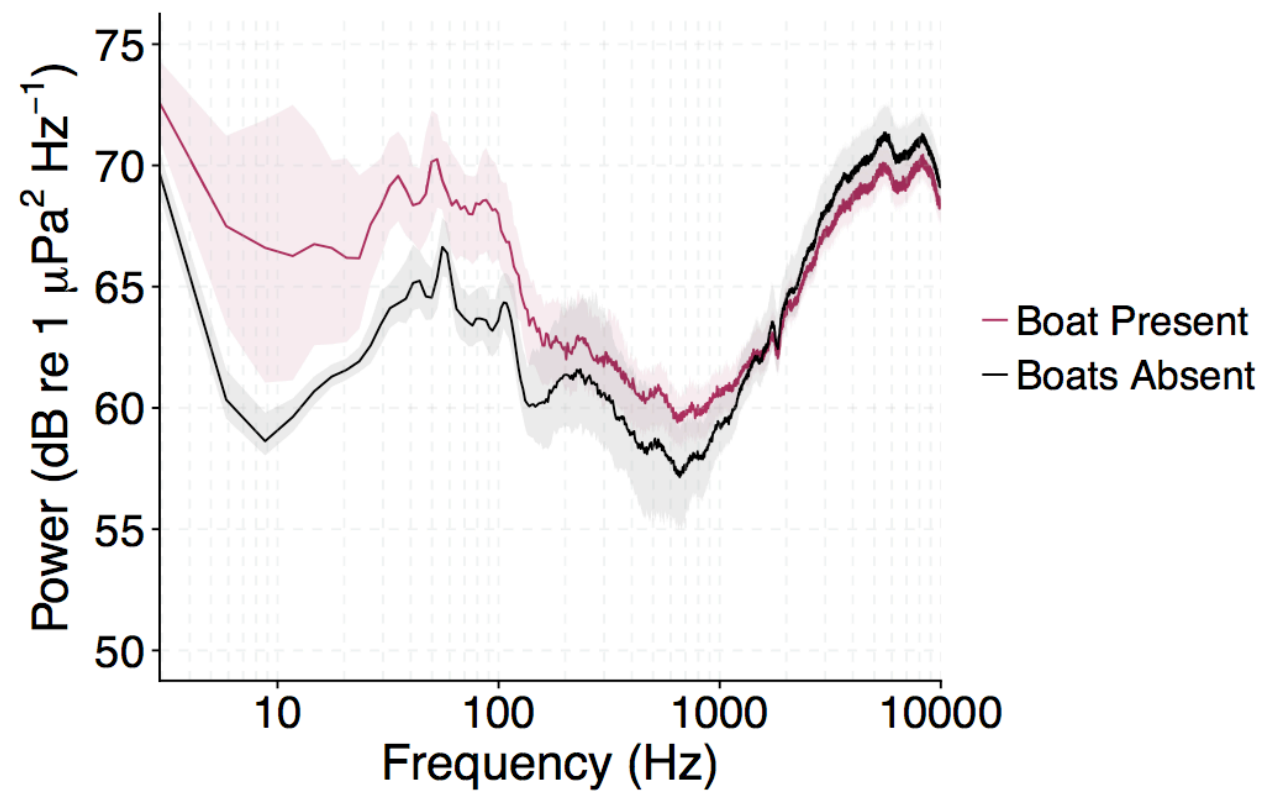


Dinh et al. 2018

\section{Figure 2: Spectral comparisons between recordings with and without boat noise.}

Spectrograms without boat noise (a) and with boat noise (b and c). Sites with boat noise show broadband elevated acoustic power in low frequencies compared to spectrograms without boat noise. (d) Average power spectra show elevated acoustic power below $1000 \mathrm{~Hz}$. Recordings with boat noise exhibit high variability in acoustic power at frequencies below $100 \mathrm{~Hz}$. Lines represent median acoustic power, while shaded areas represent $25^{\text {th }}-75^{\text {th }}$ percentiles. Average power spectra were generated from recordings taken between 3/26/2017 - 4/10/2017 at Reef 6 (n =2073). This time period and site were chosen by selecting the two first weeks of data from a randomly selected deployment at a randomly selected site. Two recordings with boat noise and one recording without boat noise were randomly selected from within this deployment. 
Dinh et al. 2018
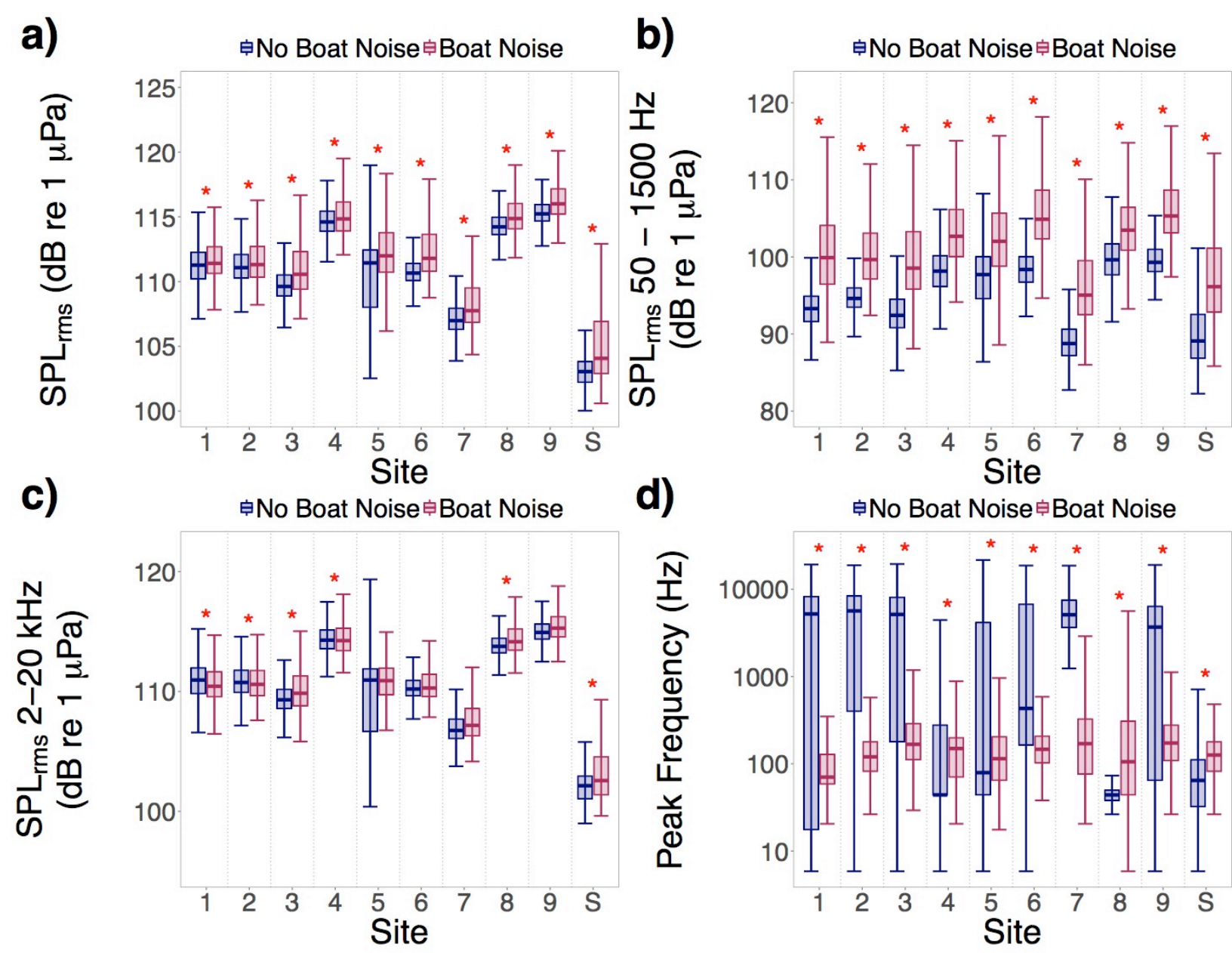

Figure 3: Acoustic effects of boat noise. Boat noise was associated with significant increases in (a) overall $\mathrm{SPL}_{\mathrm{rms}}$ and (b) low-frequency $\mathrm{SPL}_{\mathrm{rms}}$ at all sites. (c) High-frequency $\mathrm{SPL}_{\mathrm{rms}}$ were not greatly affected by boat noise despite several sites showing statistical significance. (d)

Downward shifts in median peak frequency indicate an overall change in ambient soundscapes. Thick lines indicate median values, boxes indicate $25^{\text {th }}$ and $75^{\text {th }}$ quantiles, and whiskers indicate maximal and minimal values excluding outliers. Asterisks denote statistical significance between natural noise and boat noise after the Bonferroni correction $(\mathrm{p}<0.0125)$. 
Dinh et al. 2018

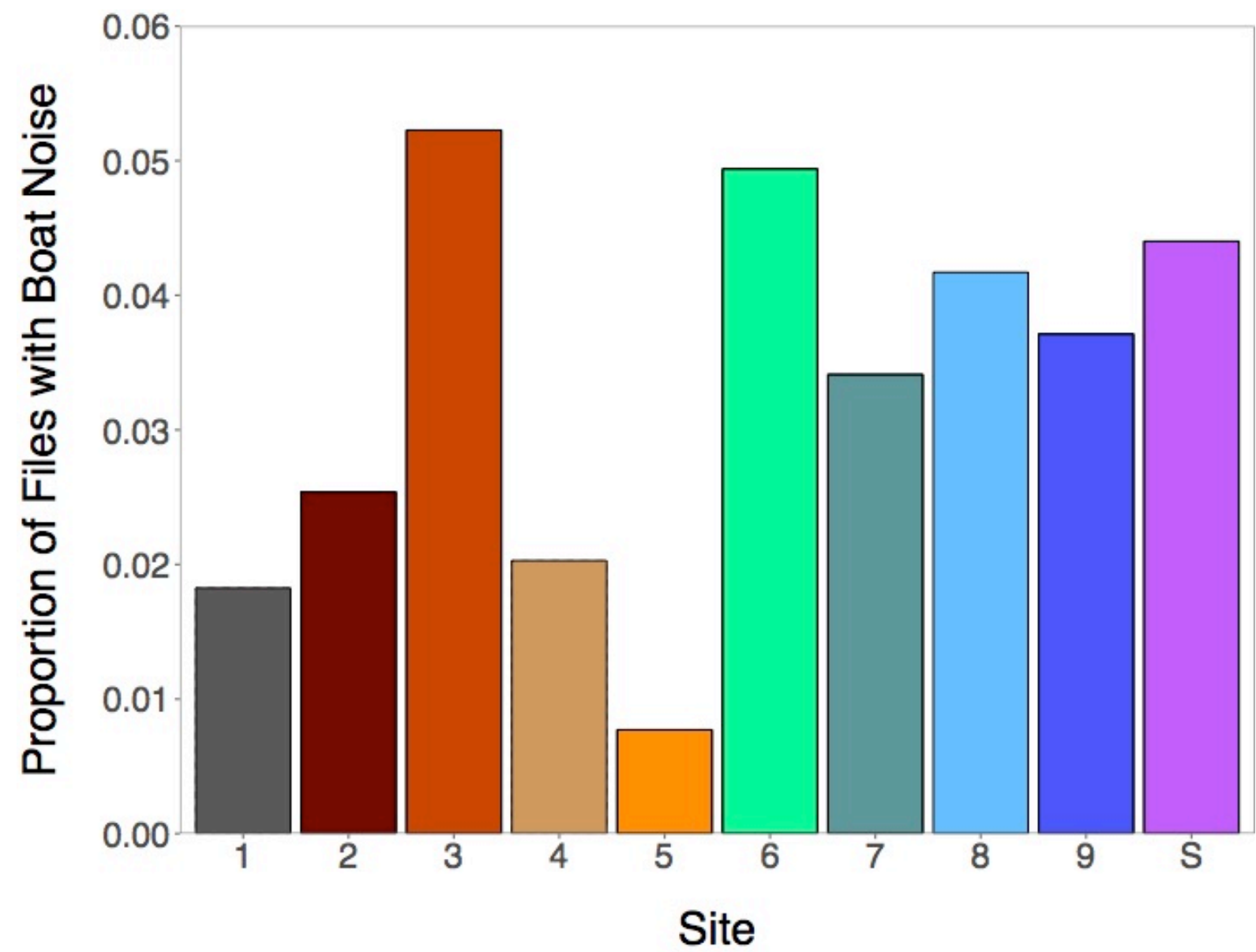

Figure 4: Variation in boat noise occurrence between sites. Between 1-5\% of the total collected recordings at a given site contained boat noise. Detections were most common at Reef 3 and least common at Reef 5. 
Dinh et al. 2018
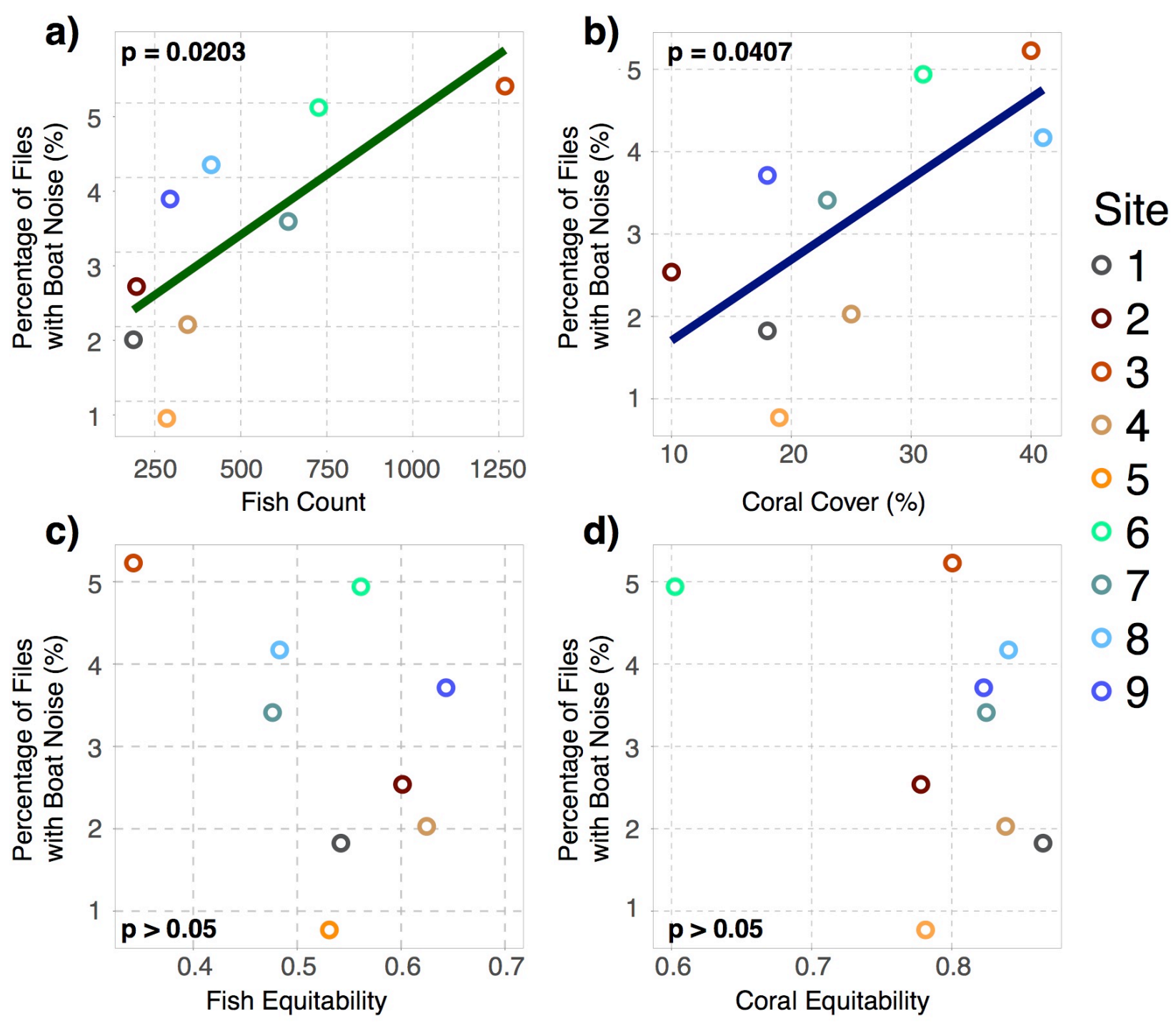

Figure 5: Ordinary least-squares regressions of boat noise occurrence versus fish and coral metrics. There was significant positive linear correlation between the percentage of files with boat noise and (a) fish density $\left(y=1.62+0.00323 * x, R^{2}=0.498, F=8.92, d f=7, p=\right.$ $0.0203)$ and (b) coral cover $\left(y=0.724+0.0983 * x, R^{2}=0.397, F=6.28, d f=7, p=\right.$ 0.0407). There was no significant correlation between the percentage of files with boat noise and (c) fish Shannon-Weiner diversity or $(d)$ coral Shannon-Weiner diversity $(p>0.05)$. The sandy shore site (S) was not included in this analysis. 

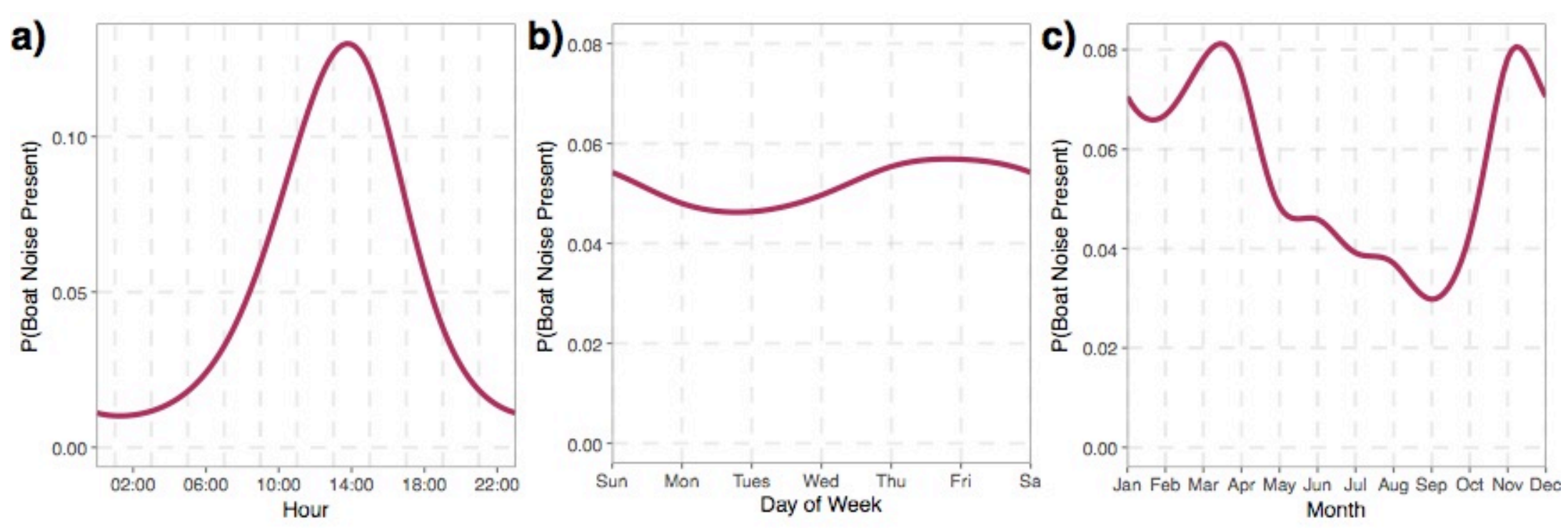

Figure 6: Smoothing estimators of GAMM fixed effects after transformation to a binary scale. Knots were capped at 4, 7, and 12 for (a) daily, (b) weekly, and (c) yearly smooths, respectively. 

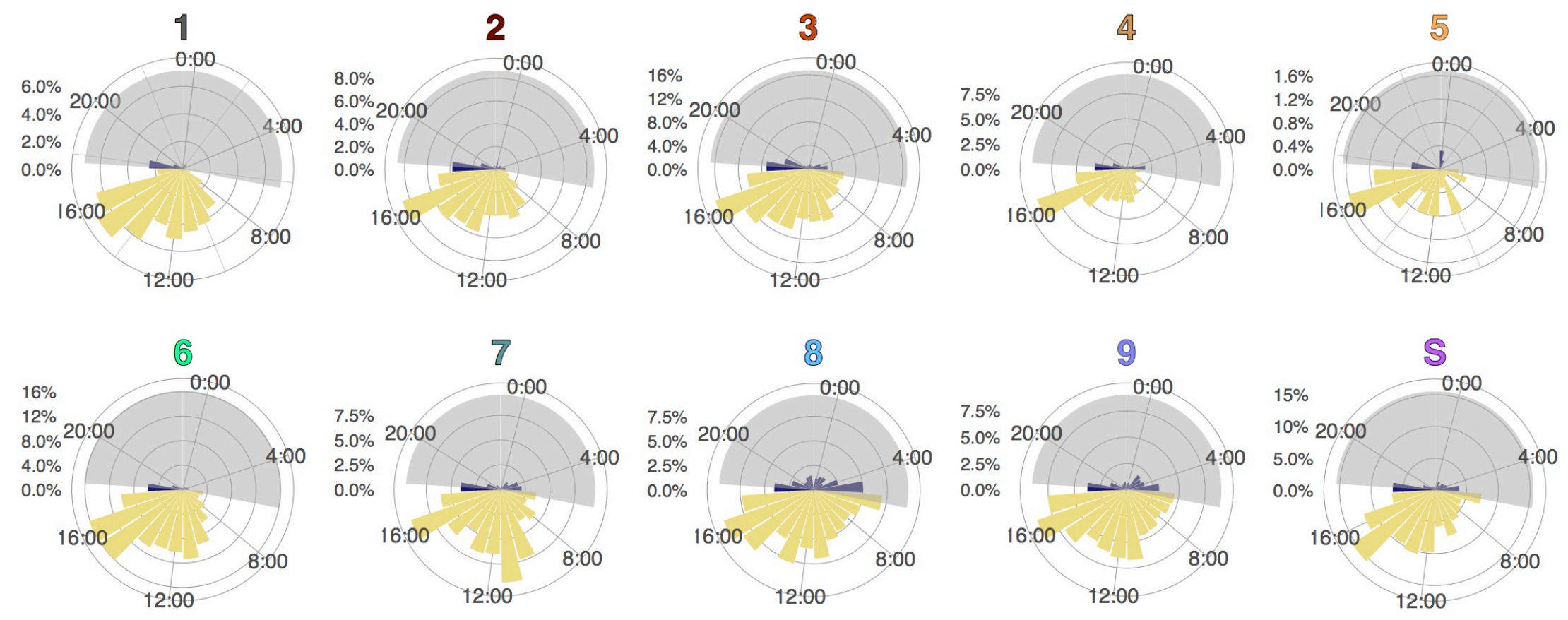

Figure 7: Diel patterns in percentage of recordings with boat noise at each site. Most sites showed low boat occurrence rates at night with a sudden increase occurring in the early daylight hours. Peaks in boat noise are observed in the late afternoon (ca 15:00).

The gold bars with a white background denote hours of daylight while the navy bars with a gray background denote hours of darkness based on winter (i.e. November - January) daylight hours. Scales vary between plots, and tick labels are noted to the left of the graph. Colors of the site numbers correspond to the coloring scheme seen in other figures. 
Dinh et al. 2018

a)

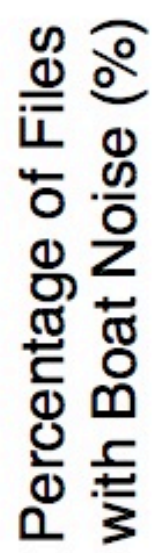

b) $\mathbb{x}$

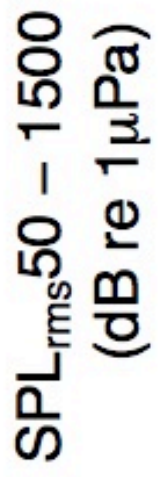

c)

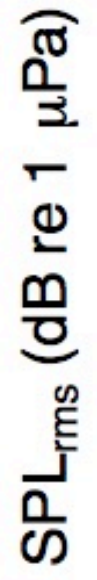

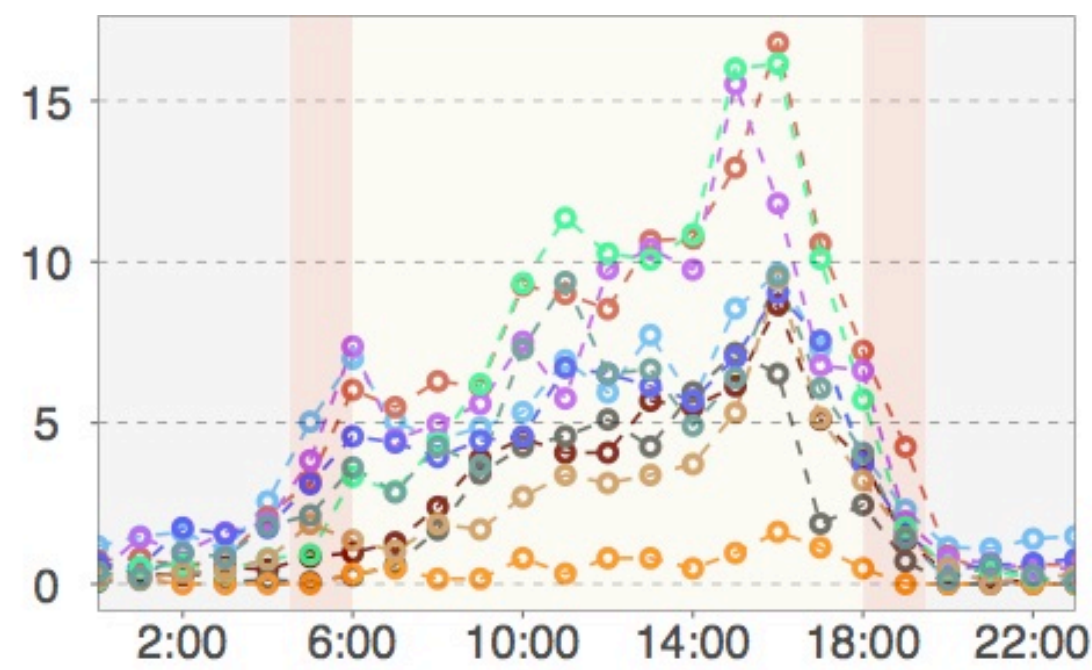

100

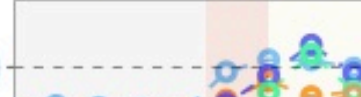

$$
10.00
$$

Site

01

0.2

0.3

.4

$\cdot 5$

$\cdot 6$

0.7

.8

0.9

$\circ S$ $\begin{array}{llllll}2: 00 & 6: 00 & 10: 00 & 14: 00 & 18: 00 & 22: 00\end{array}$

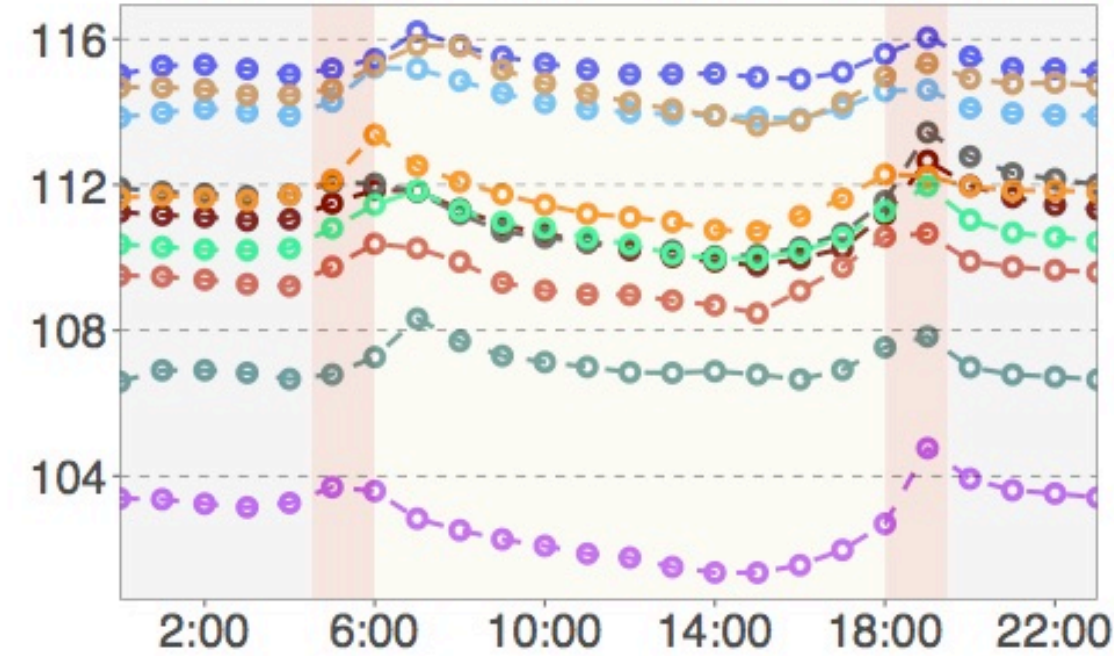

Figure 8: Diel patterns in boat noise and ambient sound levels. (a) Boat noise was lowest during nighttime hours, sharply rose at dawn, and peaked in the late afternoon. (b) Median SPL 
Dinh et al. 2018

in the low-frequency fish communication band were elevated during daytime hours, concurrent with high boating activity. (c) Median overall SPL exhibit crepuscular peaks. Only files without boat noise were selected for SPL calculations in (b) and (c), thereby eliminating the effect of boat noise in calculated sound pressure levels. Recordings were binned by hour and pooled across the entire deployment year to determine the percentage of files with boat noise and to calculate median SPL. Background colors correspond to the time of day, with light purple indicating night, red indicating dusk and dawn, and yellow indicating daylight. Daylight designations are based on winter daylight hours (November - January). 
Dinh et al. 2018

Table 1: Summary table describing the type of ecosystem, type of boat mooring present, and percentage of days with boat detections at each site. Boat noise was frequently detected at the two sites with SCUBA moorings (6 and 8) and at sites with high coral cover and fish density (see Figure 5). Generally, the sites without moorings (4, 5, and S) showed lower boat noise occurrences with the exception of Reef 3, where higher boat noise detections might be expected due to increased depth and distance offshore.

\begin{tabular}{l|l|l|l|l}
\hline Site Name & Reef Number & Ecosystem & Moorings & Percentage of Days with Boat Noise Detections \\
\hline \hline Ditliff Point & 1 & Coral Reef & Overnight & $72.10 \%$ \\
Cocoloba Cay & 2 & Coral Reef & Overnight & $84.10 \%$ \\
Joel's Shoal & 3 & Coral Reef & None & $96.80 \%$ \\
White Point & 4 & Coral Reef & None & $81.20 \%$ \\
Europa Bay & 5 & Coral Reef & None & $29.80 \%$ \\
Tektite & 6 & Coral Reef & SCUBA & $96.50 \%$ \\
Yawzi Point & 7 & Coral Reef & Overnight & $93.40 \%$ \\
Booby Rock & 8 & Coral Reef & SCUBA & $91.20 \%$ \\
Ram Head & 9 & Coral Reef & Overnight & $97.30 \%$ \\
Reef Bay & $\mathrm{S}$ & Sandy Shore & None & $85.50 \%$
\end{tabular}


Dinh et al. 2018

Table 2: Summary of two fitted GAMMs. Estimated degrees of freedom, chi-squared, and pvalues are shown for each fixed effect along with the deviance explained by each model.

\begin{tabular}{lllllll}
\hline Response & $\begin{array}{l}\text { Fixed } \\
\text { Effects }\end{array}$ & edf & $\mathbf{X}^{\mathbf{2}}$ & p-value & Random Effects & Deviance Explained \\
\hline \hline $\begin{array}{l}\text { Presence of boat } \\
\text { noise }\end{array}$ & $\begin{array}{l}\text { Day of week } \\
\text { Month }\end{array}$ & 3.674 & 230.1 & $3.54 * 10^{-15}$ & Day of week within site & $4.11 \%$ \\
\hline $\begin{array}{l}\text { Presence of boat } \\
\text { noise }\end{array}$ & Hour & 1.999 & 13302 & $<2.2^{*} 10^{-16}$ & Hour within site & $9 \%$ \\
\end{tabular}


Dinh et al. 2018

Table 3: Summary of temporal peaks in boat noise at each site. Daily peaks occurred in the late afternoon (i.e. 15:00 - 16:00) at all sites except for Reef 7. Weekly peaks varied but generally occurred on weekends (i.e. Friday - Sunday) at 7 out of 10 sites. Monthly peaks varied but most commonly occurred between January and March.

\begin{tabular}{llll}
\hline Reef & $\begin{array}{l}\text { Hour with Peak Boat } \\
\text { Frequency }\end{array}$ & $\begin{array}{l}\text { Weekday with } \\
\text { Highest Boat } \\
\text { Frequency }\end{array}$ & $\begin{array}{l}\text { Month with Peak } \\
\text { Boat Frequency }\end{array}$ \\
\hline \hline 1 & $15: 00$ & Tuesday & March \\
3 & $16: 00$ & Friday & January \\
4 & $16: 00$ & Thursday & January \\
5 & $16: 00$ & Sunday & February \\
6 & $16: 00$ & Friday & May \\
7 & $15: 00$ & Friday & April \\
8 & $11: 00$ & Thursday & March \\
9 & $16: 00$ & Sunday & January \\
\hline & $16: 00$ & Sunday & March \\
& $15: 00$ & Friday & March
\end{tabular}


Dinh et al. 2018

\section{Supplementary Material:}
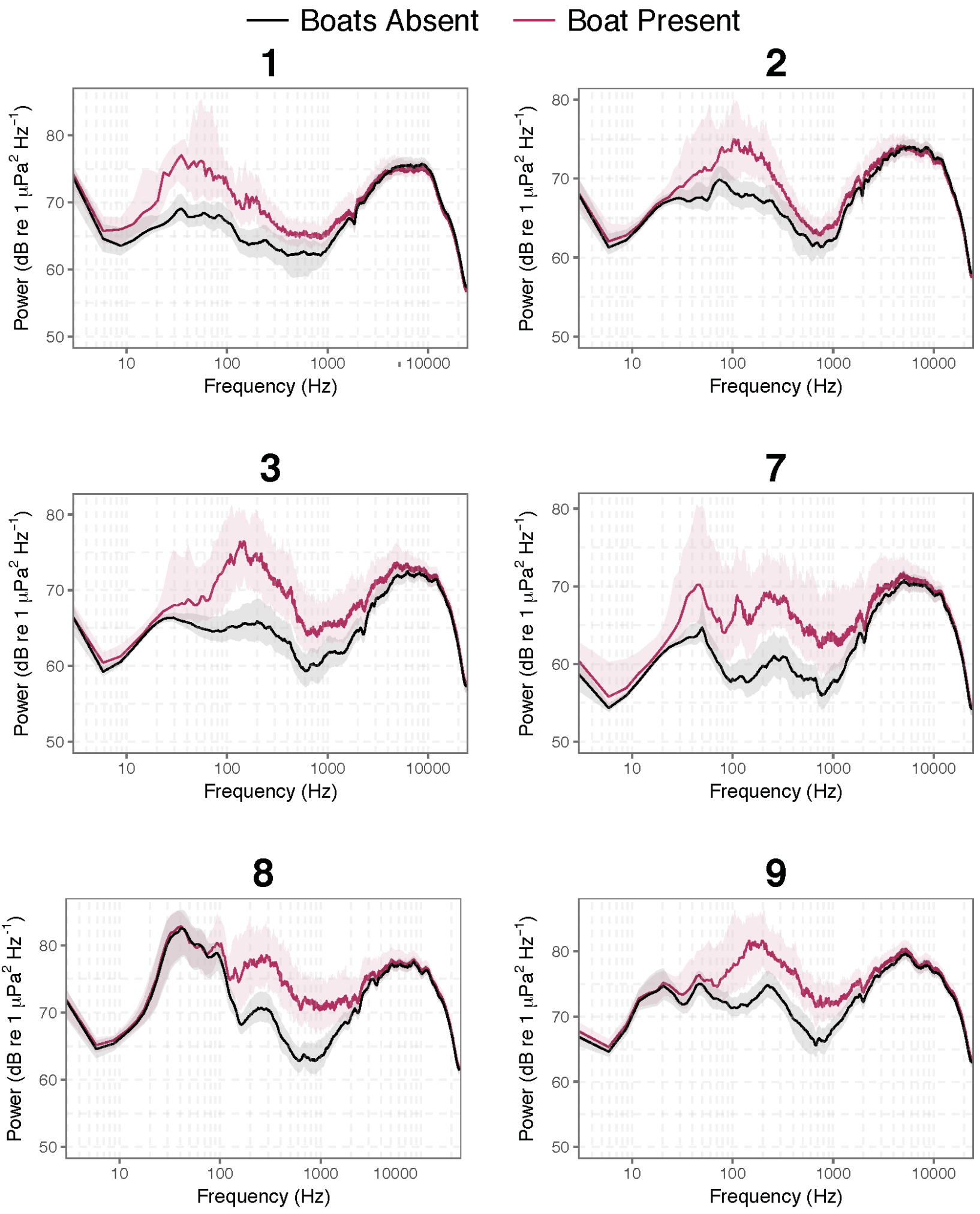
Dinh et al. 2018

Supplementary Material 1: Average power spectra at coral reef sites show elevated sound pressure levels at frequencies below $1000 \mathrm{~Hz}$ in files with boat noise. Recordings were taken from March 26 - April 10, the first two weeks of a randomly selected hydrophone deployment. Data for this time period were available for all coral reef sites except Reefs 4 and 5. 
Supplementary Material 2: Comparison of SPL and peak frequencies in recordings with and without boat noise. Medians are reported with $25^{\text {th }}-75^{\text {th }}$ percentiles. Mann-Whitney U tests were used to compare medians between files with and without boat noise within each site. Alpha was reduced to 0.0125 using the Bonferroni correction.

\begin{tabular}{|c|c|c|c|c|c|c|c|c|}
\hline \multirow{2}{*}{ Reef } & \multicolumn{4}{|c|}{ SPLrms $50-1500 \mathrm{~Hz}(\mathrm{~dB}$ re $1 \mu \mathrm{Pa})$} & \multicolumn{4}{|c|}{ Peak Frequency (Hz) } \\
\hline & Boat Absent & Boat Present & $\mathbf{U}$ & p-value & Boat Absent & Boat Present & $\mathbf{U}$ & p-value \\
\hline 1 & $93.3(91.6-94.9)$ & $100.0(96.5-104.4)$ & 46894000 & $<0.0005$ & $5210(18-8260)$ & $70(59-128)$ & 19651000 & $<0.0005$ \\
\hline 2 & $94.6(93.5-96.0)$ & 99.7 (97.2 - 103.3) & 70788000 & $<0.0005$ & $5650(400-8380)$ & $120(92-179)$ & 15391000 & $<0.0005$ \\
\hline 3 & $92.4(90.8-94.5)$ & $98.6(95.8-103.6)$ & 126780000 & $<0.0005$ & $5150(179-8100)$ & $167(111-289)$ & 41396000 & $<0.0005$ \\
\hline 4 & $98.2(96.2-100.2)$ & $102.8(100.1-106.3)$ & 28443000 & $<0.0005$ & $44(44-278)$ & $149(70-199)$ & 22085000 & $<0.0005$ \\
\hline 5 & $97.7(94.6-100.0)$ & $102.5(98.9-107.4)$ & 4422600 & $<0.0005$ & $79(44-4170)$ & $114(53-204)$ & 3070500 & 0.0397 \\
\hline 6 & $98.3(96.7-100.0)$ & $105.1(102.5-109.3)$ & 112450000 & $<0.0005$ & $431(164-6760)$ & $146(103-208)$ & 30033000 & $<0.0005$ \\
\hline 7 & $88.8(87.2-90.6)$ & $95.1(92.5-99.9)$ & 74680000 & $<0.0005$ & $5100(3640-7490)$ & $170(67-327)$ & 13611000 & $<0.0005$ \\
\hline 8 & $99.6(97.7-101.7)$ & $104(100.9-106.5)$ & 160570000 & $<0.0005$ & $44(38-50)$ & $105(44-308)$ & 132770000 & $<0.0005$ \\
\hline 9 & 99.3 (98.1 - 101.0) & $105.4(103.2-109.0)$ & 59865000 & $<0.0005$ & $3680(64-6370)$ & $173(108-278)$ & 21653000 & $<0.0005$ \\
\hline S & $89.1(86.9-92.6)$ & $96.2(92.9-101.3)$ & 28509000 & $<0.0005$ & $65(32-111)$ & $126(82-179)$ & 23875000 & $<0.0005$ \\
\hline
\end{tabular}


Dinh et al. 2018

Supplementary Material 3: Summary of collected recordings at each site. The percentage of recordings with boat noise was significantly different between sites under Pearson's chi-squared test $(\mathrm{p}<0.05)$.

\begin{tabular}{|c|c|c|c|c|}
\hline Reef & $\begin{array}{l}\text { Number of } \\
\text { Recordings with } \\
\text { Boat Noise } \\
\end{array}$ & $\begin{array}{l}\text { Total Number of } \\
\text { Recordings }\end{array}$ & $\begin{array}{l}\text { Percent of Recordings } \\
\text { with Boat Noise }\end{array}$ & $\begin{array}{l}\text { Percent of Days with } \\
\text { Boat Detections }\end{array}$ \\
\hline 1 & 990 & 54249 & $1.82 \%$ & $72.1 \%$ \\
\hline 2 & 1442 & 56805 & $2.54 \%$ & $84.1 \%$ \\
\hline 3 & 2830 & 54147 & $5.23 \%$ & $96.8 \%$ \\
\hline 4 & 848 & 41798 & $2.03 \%$ & $81.2 \%$ \\
\hline 5 & 210 & 27231 & $0.771 \%$ & $29.8 \%$ \\
\hline 6 & 2500 & 50617 & $4.94 \%$ & $96.5 \%$ \\
\hline 7 & 1703 & 49923 & $3.41 \%$ & $93.4 \%$ \\
\hline 8 & 3000 & 71928 & $4.17 \%$ & $91.2 \%$ \\
\hline 9 & 1586 & 42723 & $3.70 \%$ & $97.3 \%$ \\
\hline $\mathrm{S}$ & 1250 & 28406 & $4.40 \%$ & $85.5 \%$ \\
\hline
\end{tabular}


Dinh et al. 2018

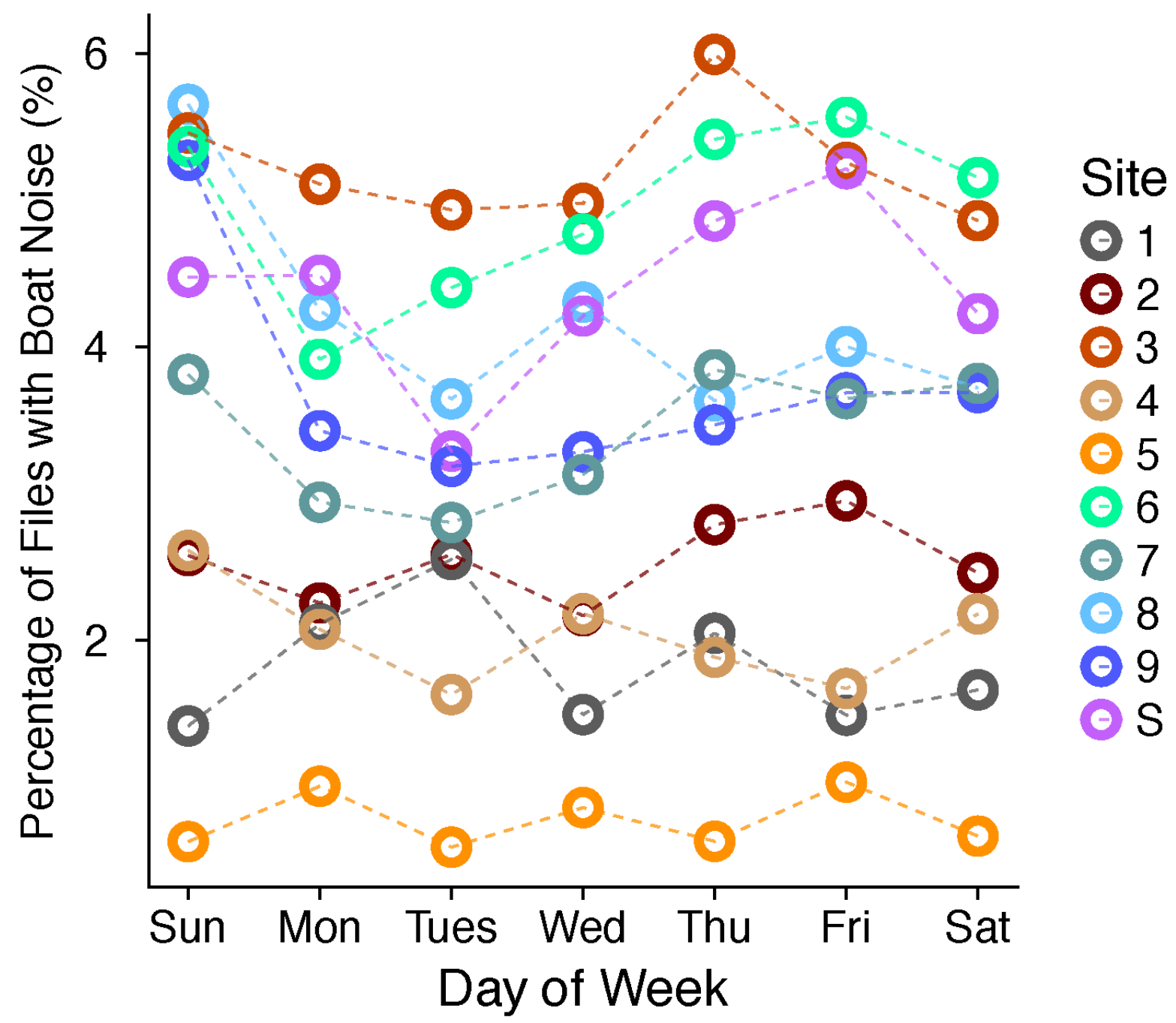

Supplementary Material 4: Weekly patterns in percentage of files with boat noise. Weekly patterns in boat noise are less consistent across sites than daily and annual patterns. Most sites showed peaks in boat detections between Thursday and Sunday, with lower detection rates early in the week. 
Dinh et al. 2018

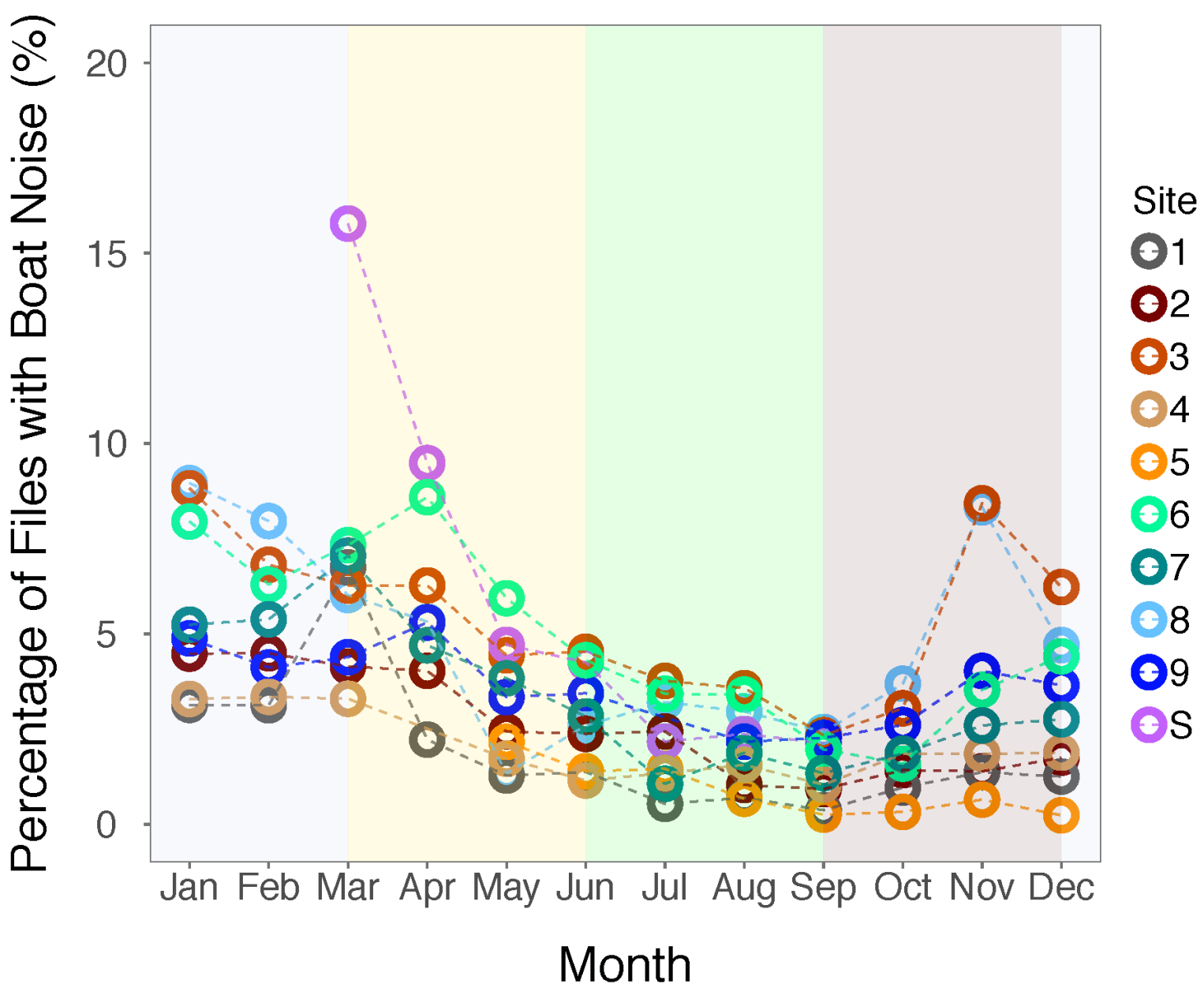

Supplementary Material 5: Monthly variation in boat noise at each monitored site. Background colors indicate meteorological seasons. Boats are most prevalent in the winter months and least prevalent in the summer months. Peak months vary between sites. However, all sites show maximum boat detections between January and March and minimum boat detections between June and August. Data are missing from the following sites due to equipment malfunction: Reef 4 (March 29 - June 19, 2017), Reef 5 (December 12, 2016 - June 19, 2017), , Reef 9 (June 29 August 7, 2016), and Site S (October 24, 2016 - March 28, 2017). High boat noise occurrence rates at Site $\mathrm{S}$ in March is due to low sample size $(\mathrm{n}=63)$. 